\title{
Resolving dominating partitions in graphs
}

\author{
Carmen Hernando*1, Mercè Mora ${ }^{\dagger 1}$, and Ignacio M. Pelayo ${ }^{\ddagger 1}$ \\ ${ }^{1}$ Departament de Matemàtiques, Universitat Politècnica de Catalunya
}

\begin{abstract}
A partition $\Pi=\left\{S_{1}, \ldots, S_{k}\right\}$ of the vertex set of a connected graph $G$ is called a resolving partition of $G$ if for every pair of vertices $u$ and $v, d\left(u, S_{j}\right) \neq d\left(v, S_{j}\right)$, for some part $S_{j}$. The partition dimension $\beta_{p}(G)$ is the minimum cardinality of a resolving partition of $G$. A resolving partition $\Pi$ is called resolving dominating if for every vertex $v$ of $G, d\left(v, S_{j}\right)=1$, for some part $S_{j}$ of $\Pi$. The dominating partition dimension $\eta_{p}(G)$ is the minimum cardinality of a resolving dominating partition of $G$.

In this paper we show, among other results, that $\beta_{p}(G) \leq \eta_{p}(G) \leq \beta_{p}(G)+1$. We also characterize all connected graphs of order $n \geq 7$ satisfying any of the following conditions: $\eta_{p}(G)=n, \eta_{p}(G)=n-1, \eta_{p}(G)=n-2$ and $\beta_{p}(G)=n-2$. Finally, we present some tight Nordhaus-Gaddum bounds for both the partition dimension $\beta_{p}(G)$ and the dominating partition dimension $\eta_{p}(G)$.
\end{abstract}

Keywords: resolving partition, resolving dominating partition, metric location, resolving domination, partition dimension, dominating partition dimension.

AMS subject classification: 05C12, 05C35, 05C69.

\section{Introduction}

Domination and location in graphs are two important subjects that have received a high attention in the literature, usually separately, but sometimes also both together. These concepts are useful to distinguish the vertices of a graph in terms of distances to a given set of vertices or by considering their neighbors in this set. Resolving sets were introduced independently in the 1970s by Slater [26], as locating sets, and by Harary and Melter [14], whereas dominating sets were defined in the 1960s by Ore [21]. Both types of sets have many and varied applications in other areas. For example, resolving sets have applications in robot navigation [26], combinatorial optimization [25], game theory [10], pharmaceutical chemistry [5] and in other contexts [2,4]. On the other hand, dominating sets are helpful to design and analyze communication networks $[8,24]$ and to model biological networks [16].

*Partially supported by projects MTM2015-63791-R (MINECO/FEDER) and Gen.Cat. DGR2017SGR1336, carmen.hernando@upc.edu

${ }^{\dagger}$ Partially supported by projects MTM2015-63791-R (MINECO/FEDER), Gen.Cat. DGR2017SGR1336 and H2020-MSCA-RISE-2016-734922 CONNECT, merce.mora@upc.edu

${ }^{\ddagger}$ Partially supported by project MINECO MTM2014-60127-P, ignacio.m.pelayo@upc.edu 
Many variations of location in graphs have since been defined (see survey [23]). For example, in 2000, Chartrand, Salehi and Zhang study the resolvability of graphs in terms of partitions [6], as a generalization of resolving sets when the vertices are classified in different types. A few years later, resolving dominating sets were introduced by Brigham, Chartrand, Dutton and Zhang [1] and independently by Henning and Oellermann [17] as metric-locating-dominating sets, combining the usefulness of resolving sets and dominating sets. Resolving dominating sets have been further studied in $[3,11,19]$. In this paper, following the ideas of these works, we introduce the resolving dominating partitions, as a way for distinguishing the vertices of a graph by using on the one hand partitions, and on the other hand, both domination and location.

\subsection{Basic terminology}

All the graphs considered are undirected, simple, finite and (unless otherwise stated) connected. Let $v$ be a vertex of a graph $G$. The open neighborhood of $v$ is $N_{G}(v)=\{w \in V(G): v w \in E\}$, and the closed neighborhood of $v$ is $N_{G}[v]=N_{G}(v) \cup\{v\}$ (we will write $N(v)$ and $N[v]$ if the graph $G$ is clear from the context). The degree of $v$ is $\operatorname{deg}(v)=|N(v)|$. The minimum degree (resp. maximum degree) of $G$ is $\delta(G)=\min \{\operatorname{deg}(u): u \in V(G)\}$ (resp. $\Delta(G)=\max \{\operatorname{deg}(u): u \in V(G)\}$ ). If $\operatorname{deg}(v)=1$, then $v$ is said to be a leaf of $G$.

The distance between vertices $v, w \in V(G)$ is denoted by $d_{G}(v, w)$, or $d(v, w)$ if the graph $G$ is clear from the context. The diameter of $G$ is $\operatorname{diam}(G)=\max \{d(v, w): v, w \in V(G)\}$. The distance between a vertex $v \in V(G)$ and a set of vertices $S \subseteq V(G)$, denoted by $d(v, S)$, is the minimum of the distances between $v$ and the vertices of $S$, that is, $d(v, S)=\min \{d(v, w): w \in S\}$.

Let $u, v \in V(G)$ be a pair of vertices such that $d(u, w)=d(v, w)$ for all $w \in V(G) \backslash\{u, v\}$, i.e., such that either $N(u)=N(v)$ or $N[u]=N[v]$. In both cases, $u$ and $v$ are said to be twins. Let $W$ be a set of vertices of $G$. If the vertices of $W$ are pairwise twins, then $W$ is called a twin set of $G$.

Let $W \subseteq V(G)$ be a subset of vertices of $G$. The closed neighborhood of $W$ is $N[W]=\cup_{v \in W} N[v]$. The subgraph of $G$ induced by $W$, denoted by $G[W]$, has $W$ as vertex set and $E(G[W])=\{v w \in$ $E(G): v \in W, w \in W\}$.

The complement of $G$, denoted by $\bar{G}$, is the graph on the same vertices as $G$ such that two vertices are adjacent in $\bar{G}$ if and only if they are not adjacent in $G$. Let $G_{1}, G_{2}$ be two graphs having disjoint vertex sets. The (disjoint) union $G=G_{1}+G_{2}$ is the graph such that $V(G)=V\left(G_{1}\right) \cup V\left(G_{2}\right)$ and $E(G)=E\left(G_{1}\right) \cup E\left(G_{2}\right)$. The join $G=G_{1} \vee G_{2}$ is the graph such that $V(G)=V\left(G_{1}\right) \cup V\left(G_{2}\right)$ and $E(G)=E\left(G_{1}\right) \cup E\left(G_{2}\right) \cup\left\{u v: u \in V\left(G_{1}\right), v \in V\left(G_{2}\right)\right\}$.

\subsection{Metric dimension and partition dimension}

A set of vertices $S \subseteq V(G)$ of a graph $G$ is a resolving set of $G$ if for every pair of distinct vertices $v, w \in V(G), d(v, x) \neq d(w, x)$, for some vertex $x \in S$. The metric dimension $\beta(G)$ of $G$ is the minimum cardinality of a resolving set.

Resolving sets were introduced independently in papers [14] and [26] (in this last work they were called locating sets), and since then they have been widely investigated (see [4, 18, 25] and their references).

Let $\Pi=\left\{S_{1}, \ldots, S_{k}\right\}$ be a partition of $V(G)$. We denote by $r(u \mid \Pi)$ the vector of distances between a vertex $u \in V(G)$ and the elements of $\Pi$, that is, $r(u \mid \Pi)=\left(d\left(u, S_{1}\right), \ldots, d\left(u, S_{k}\right)\right)$. If $u, v \in V(G)$, we say that a part $S_{i}$ of $\Pi$ resolves $u$ and $v$ if $d\left(u, S_{i}\right) \neq d\left(v, S_{i}\right)$. If $V^{\prime} \subseteq V(G)$, we say that a part $S_{i}$ of $\Pi$ resolves $V^{\prime}$ if $S_{i}$ resolves every pair of vertices of $V^{\prime}$. 
A partition $\Pi=\left\{S_{1}, \ldots, S_{k}\right\}$ is called a resolving partition of $G$ if for any pair of distinct vertices $u, v \in V(G), r(u \mid \Pi) \neq r(v \mid \Pi)$, that is, if the set $\{u, v\}$ is resolved by some part $S_{i}$ of $\Pi$.

The partition dimension $\beta_{p}(G)$ of $G$ is the minimum cardinality of a resolving partition of $G$. Resolving partitions were introduced in [6], and further studied in $[7,9,12,13,22,28]$. In some of these papers the partition dimension of $G$ is denoted by $p d(G)$. Next, some known results concerning this parameter are shown.

Theorem 1 ([6]). Let $G$ be a graph of order $n \geq 2$. Then,

(1) $\beta_{p}(G) \leq \beta(G)+1$.

(2) $\beta_{p}(G) \leq n-\operatorname{diam}(G)+1$. Moreover, this bound is sharp.

(3) $\beta_{p}(G)=2$ if and only if $G$ is isomorphic to the path $P_{n}$.

(4) $\beta_{p}(G)=n$ if and only if $G$ is isomorphic to the complete graph $K_{n}$.

(5) If $n \geq 6$, then $\beta_{p}(G)=n-1$ if and only if $G$ is isomorphic to either the star $K_{1, n-1}$, or the complete split graph $K_{n-2} \vee \overline{K_{2}}$, or the graph $K_{1} \vee\left(K_{1}+K_{n-2}\right)$.

Remark 2. Notice that the restriction $n \geq 6$ of Theorem 1(5) is tight, since $\beta_{p}\left(C_{4}\right)=3$ and $\beta_{p}\left(C_{4} \vee K_{1}\right)=4$. Thus, in [6], the condition $n \geq 3$ of Theorem 3.3 is incorrect.

Proposition $3([7])$. Given a pair of integers $a, b$ such that $3 \leq a \leq b+1$, there exists a graph $G$ with $\beta_{p}(G)=a$ and $\beta(G)=b$.

The remaining part of this paper is organized as follows. In Section 2, we introduce the dominating partition number $\eta_{p}(G)$ and show some basic properties for this new parameter. Finally, Section 3 is devoted to the characterization of all graphs $G$ satisfying any of the following conditions: $\eta_{p}(G)=n, \eta_{p}(G)=n-1, \eta_{p}(G)=n-2$ and $\beta_{p}(G)=n-2$ and to show some tight NordhausGaddum bounds for both the partition dimension $\beta_{p}(G)$ and the dominating partition dimension $\eta_{p}(G)$.

\section{Dominating partition dimension}

A set $D$ of vertices of a graph $G$ is a dominating set if $d(v, D)=1$, for every vertex $v \in V(G) \backslash D$. The domination number $\gamma(G)$ is the minimum cardinality of a dominating set.

A set $S \subseteq V(G)$ is a resolving dominating set, if it is both resolving and dominating. The resolving domination number $\eta(G)$ of $G$ is the minimum cardinality of a resolving dominating set of $G$. Resolving dominating sets were introduced in [1], and also independently in [17] (in this last work they were called metric-locating-dominating sets), being further studied in [3, 11, 15, 19, 20, 27].

As a straightforward consequence of these definitions, it holds that (see [3]):

$$
\max \{\gamma(G), \beta(G)\} \leq \eta(G) \leq \gamma(G)+\beta(G) .
$$

A partition $\Pi=\left\{S_{1}, \ldots, S_{k}\right\}$ of $V(G)$ is called dominating if for every $v \in V(G), d\left(v, S_{j}\right)=1$ for some $j \in\{1, \ldots, k\}$. The partition domination number $\gamma_{p}(G)$ equals the minimum cardinality of a dominating partition in $G$. 
Proposition 4. For any non-trivial graph $G, \gamma_{p}(G)=2$.

Proof. Let $S$ be a dominating set of cardinality $\gamma(G)$. Observe that the partition $\Pi=\{S, V(G) \backslash S\}$ is a dominating partition of $G$. Hence, $\gamma_{p}(G)=2$, since $G$ is non-trivial.

Let $\Pi=\left\{S_{1}, \ldots, S_{k}\right\}$ be a partition of the vertex set of a non-trivial graph $G$. The partition $\Pi$ is called a resolving dominating partition of $G, R D$-partition for short, if it is both resolving and dominating. The dominating partition dimension $\eta_{p}(G)$ of $G$ is the minimum cardinality of an RD-partition of $G$. An RD-partition of cardinality $\eta_{p}(G)$ is called an $\eta_{p}(G)$-partition of $G$.

Proposition 5. If $G$ is a non-trivial graph, then $\eta_{p}(G)=2$ if and only if $G$ is isomorphic to $K_{2}$.

Proof. Certainly, $\eta_{p}\left(K_{2}\right)=2$. Conversely, let $G$ be a graph such that $\eta_{p}(G)=2$. Take an $\eta_{p}(G)$ partition $\Pi=\left\{S_{1}, S_{2}\right\}$. Suppose that for some $i \in\{1,2\},\left|S_{i}\right| \geq 2$. Assume w.l.o.g. that $i=1$ and take $u, v \in S_{1}$. As $\Pi$ is a dominating partition, $r(u \mid \Pi)=(0,1)=r(v \mid \Pi)$, contradicting that $\Pi$ is a resolving partition. So, $\left|S_{1}\right|=\left|S_{2}\right|=1$ and thus $G \cong K_{2}$.

Proposition 6. Let $P_{n}$ and $C_{n}$ denote the path and the cycle of order $n$, respectively. If $n \geq 3$, then $\eta_{p}\left(P_{n}\right)=\eta_{p}\left(C_{n}\right)=3$.

Proof. According to Proposition 5, it is sufficient to show, in both cases, the existence of an RDpartition of cardinality 3. Assume that $V=V\left(P_{n}\right)=V\left(C_{n}\right)=\{1, \ldots, n\} ; E\left(P_{n}\right)=\{\{i, i+1\}: 1 \leq$ $i<n\}$ and $E\left(C_{n}\right)=E\left(P_{n}\right) \cup\{\{1, n\}\}$. Consider the following sets of vertices:

$S_{1}=\{1\}, S_{1}^{\prime}=\{1,2\}, S_{2}=\{i \in V: i$ even $\}, S_{2}^{\prime}=\{i \in V: i \neq 2$, even $\}, S_{3}=\{i \in V: i \neq 1$, odd $\}$.

It is straightforward to check that $\Pi=\left\{S_{1}, S_{2}, S_{3}\right\}$ is an RD-partition of $P_{n}$, and also of $C_{n}$ if $n$ is odd, and that $\Pi^{\prime}=\left\{S_{1}^{\prime}, S_{2}^{\prime}, S_{3}\right\}$ is an RD-partition of $C_{n}$, if $n$ is even.

Next, we show some results relating the dominating partition dimension $\eta_{p}$ to other parameters such as the resolving domination number $\eta$, the partition dimension $\beta_{p}$, the order and the diameter.

Proposition 7. For any graph $G$ of order $n \geq 2, \eta_{p}(G) \leq \eta(G)+1$.

Proof. Suppose that $\eta(G)=k$. Notice that $k \leq n-1$, since $n \geq 2$. Let $S=\left\{u_{1}, \ldots, u_{k}\right\}$ be a resolving dominating set of $G$. Then, $\Pi=\left\{\left\{u_{1}\right\}, \ldots,\left\{u_{k}\right\}, V(G) \backslash S\right\}$ is an RD-partition of $G$.

Lemma 8. Let $G$ be a graph of order $n \geq 3$. Let $W \subsetneq V(G)$ be a twin set of cardinality $k \geq 2$.

(1) If $W$ induces an empty graph, then $\eta_{p}(G) \geq \beta_{p}(G) \geq k$.

(2) If $W$ induces a complete graph, then $\eta_{p}(G) \geq \beta_{p}(G) \geq k+1$.

(3) If $W$ is a set of leaves, then $\eta_{p}(G) \geq k+1$.

Proof. (1) Let $W$ be a twin set of cardinality $k$. Since $d\left(w_{1}, v\right)=d\left(w_{2}, v\right)$ for every $w_{1}, w_{2} \in W$ and for every $v \in V(G) \backslash\left\{w_{1}, w_{2}\right\}$, we have that different vertices of $W$ must belong to different parts of any resolving partition. Hence, $\eta_{p}(G) \geq \beta_{p}(G) \geq k$.

Observe that, if $\beta_{p}(G)=k$, then every part of a resolving partition of cardinality $k$ contains exactly one vertex of $W$. 
(2) Suppose that $W$ induces a complete graph and $W \subsetneq V(G)$. Since $G$ is connected, there exists a vertex $v$ adjacent to all the vertices of $W$. If $\beta_{p}(G)=k$ and $\Pi$ is a resolving partition of cardinality $k$, then there is some vertex $w \in W$ such that $v$ and $w$ belong to the same part $S$ of $\Pi$. Then, $v$ and $w$ are at distance 1 from any part of $\Pi$ different from $S$, implying that $r(v \mid \Pi)=r(w \mid \Pi)$, a contradiction. Therefore, $\beta_{p}(G) \geq k+1$.

(3) Assume that $W$ is a twin set of leaves hanging from a vertex $u$. Suppose that $\eta_{p}(G)=k$ and $\Pi$ is an RD-partition of cardinality $k$. Then, $\Pi$ is also a resolving partition of cardinality $k$. Hence, there is some vertex $w \in W$ such that $u$ and $w$ belong to the same part $S$ of $\Pi$. But in such a case, $\Pi$ is not a dominating partition, because $w$ is a leaf hanging from $u$. Therefore, $\eta_{p}(G) \geq k+1$.

Proposition 9. Given a pair of integers $a, b$ such that $3 \leq a \leq b+1$, there exists a graph $G$ with $\eta_{p}(G)=a$ and $\eta(G)=b$.

Proof. Let $h=a-2$ and $k=b-a+2$. Take the caterpillar $G$ of order $n=2 k+h$ displayed in Figure 1. The set $W=\left\{w_{1}, \ldots, w_{h}, u_{1}\right\}$ is a twin set of $h+1$ leaves. Thus, by Lemma 8 , we have $\eta_{p}(G) \geq h+2$. Now, take the partition $\Pi=\left\{\left\{u_{1}, \ldots, u_{k}\right\},\left\{v_{1}, \ldots, v_{k}\right\},\left\{w_{1}\right\}, \ldots,\left\{w_{h}\right\}\right\}$. Clearly, $\Pi$ is both a dominating and a resolving partition. Hence, $\eta_{p}(G)=h+2=a$.

To prove that $\eta(G)=b$, note first that every resolving dominating set $S$ must contain all vertices from the twin set $W$ except at most one. Observe also that for every $i \in\{1, \ldots, k\}$, either $u_{i}$ or $v_{i}$ must belong to $S$. Thus, $\eta(G) \geq h+k=b$. Now, take the set $S=\left\{w_{1}, \ldots, w_{h}, u_{1}, \ldots, u_{k}\right\}$. Clearly, $S$ is both dominating and resolving. Hence, $\eta(G)=h+k=b$.

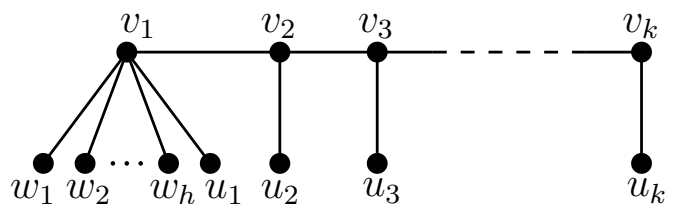

Figure 1: Caterpillar $G$ of order $n=2 k+h, \eta_{p}(G)=h+2$ and $\eta(G)=h+k$.

Next, a remarkable double inequality relating both the partition dimension and the dominating partition dimension is shown.

Theorem 10. For any graph $G$ of order $n \geq 3, \beta_{p}(G) \leq \eta_{p}(G) \leq \beta_{p}(G)+1$.

Proof. The first inequality follows directly from the definition of RD-partition. Let $\beta_{p}(G)=r$ and let $\Pi=\left\{S_{1}, \ldots, S_{r}\right\}$ be a resolving partition of $G$. If $\Pi$ is a dominating partition, then $\eta_{p}(G)=\beta_{p}(G)$. Suppose that $\Pi$ is not a dominating partition. Let $W=\left\{u \in V(G): N[u] \subseteq S_{i}\right.$ for some $i \in$ $\{1, \ldots, r\}\}$. Note that $W \neq \emptyset$, since $\Pi$ is not dominating, and that $S_{i} \backslash W \neq \emptyset$ for every $i \in\{1, \ldots, r\}$, since $G$ is connected. In order to show that $\eta_{p}(G) \leq \beta_{p}(G)+1$, we construct an RD-partition of cardinality $r+1$.

Let $C_{1}, \ldots, C_{s}$ be the connected components of the subgraph $G[W]$ induced by $W$. Clearly, for every $i \in\{1, \ldots, s\}$, all vertices of $C_{i}$ belong to the same part of $\Pi$. Next, we define a subset $W^{\prime} \subseteq W$ 
as follows. If $\left|V\left(C_{i}\right)\right|=1$, then add to $W^{\prime}$ the unique vertex of $C_{i}$. If $\left|V\left(C_{i}\right)\right| \geq 2$, then consider a 2-coloring of a spanning tree of $C_{i}$, choose one color and add to $W^{\prime}$ all vertices having this color. Note that, if $V\left(C_{k}\right) \subseteq S_{i_{k}}$ and a pair of vertices $x, y \in C_{k}$ are adjacent, then one endpoint of $x y$ is in $W^{\prime} \cup S_{i_{k}}$ and the other one belongs to $S_{i_{k}} \backslash W^{\prime}$. Let $\Pi^{\prime}=\left\{S_{1}^{\prime}, \ldots, S_{r}^{\prime}, W^{\prime}\right\}$, where $S_{i}^{\prime}=S_{i} \backslash W^{\prime} \subseteq S_{i}$ for every $1 \leq i \leq r$. We claim that $\Pi^{\prime}$ is an RD-partition.

On the one hand, observe that the sets $S_{1}^{\prime}, \ldots, S_{r}^{\prime}, W^{\prime}$ are nonempty by construction. On the other hand, notice that for every $u \in S_{i}, d\left(u, S_{j}\right)=d(u, w)$ for some vertex $w \in S_{j} \backslash W$ whenever $i \neq j$. Indeed, assume to the contrary that $d\left(u, S_{j}\right)=d(u, w)$ and $w \in S_{j} \cap W$. Since $w \in W$, we have $N[w] \subseteq S_{j}$. Thus, the vertex $w^{\prime}$ adjacent to $w$ in a shortest $(u, w)$-path is also in $S_{j}$, implying that $d\left(u, S_{j}\right) \leq d\left(u, w^{\prime}\right)<d(u, w)=d\left(u, S_{j}\right)$, a contradiction. From this last observation, we conclude that $d\left(u, S_{j}\right)=d\left(u, S_{j}^{\prime}\right)$ if $u \in S_{i}$ and $j \neq i$.

Next, we show that $\Pi^{\prime}$ is a dominating partition, i.e., that for any $u \in V(G)$, the vector $r\left(u \mid \Pi^{\prime}\right)$ has at least one component equal to 1 . We distinguish two cases.

Case 1: $u \in W^{\prime}$. Assume that $u \in S_{i}$, for some $i \in\{1, \ldots, r\}$. If $u$ belongs to a trivial connected component of $G[W]$, then every neighbor of $u$ is in $S_{i}^{\prime}$. So, $d\left(u, S_{i}^{\prime}\right)=1$. If $u$ belongs to a non-trivial connected component $C_{k}$ of $G[W]$, then any neighbor of $u$ with different color in the spanning tree of $C_{k}$ considered in the construction of $W^{\prime}$ belongs to $S_{i}^{\prime}$. So, $d\left(u, S_{i}^{\prime}\right)=1$.

Case 2: $u \in S_{i}^{\prime}$, for some $i \in\{1, \ldots, r\}$. If $u \notin W$, as $u \in S_{i}^{\prime} \backslash W=S_{i} \backslash W$, then $u$ has a neighbor $v$ in some $S_{j}$ with $j \neq i$. Therefore, $d\left(u, S_{j}^{\prime}\right)=1$ if $v \in S_{j}^{\prime}$, and $d\left(u, W^{\prime}\right)=1$ if $v \in W^{\prime}$. If $u \in W$, then $u$ belongs to a non-trivial connected component of $G[W]$ and, by construction of $W^{\prime}, u$ has a neighbor in $W^{\prime}$. Thus, $d\left(u, W^{\prime}\right)=1$.

Finally, we show that $\Pi^{\prime}$ is a resolving partition, i.e., that $r\left(u \mid \Pi^{\prime}\right) \neq r\left(v \mid \Pi^{\prime}\right)$ for every pair of distinct vertices $u, v \in V(G)$ belonging to the same part of $\Pi^{\prime}$. We distinguish two cases.

Case 1: $u, v \in S_{i}^{\prime}$ for some $i \in\{1, \ldots, r\}$. In such a case, $u, v \in S_{i}$. Since $\Pi$ is a resolving partition, $d\left(u, S_{j}\right) \neq d\left(v, S_{j}\right)$ for some $j \neq i$. Using the observation above, we have that $d\left(u, S_{j}^{\prime}\right)=d\left(u, S_{j}\right) \neq$ $d\left(v, S_{j}\right)=d\left(v, S_{j}^{\prime}\right)$ for some $j \neq i$. Therefore, $r\left(u \mid \Pi^{\prime}\right) \neq r\left(v \mid \Pi^{\prime}\right)$.

Case 2: $u, v \in W^{\prime}$. If $u, v \in S_{i}$ for some $i \in\{1, \ldots, r\}$, then proceeding as in the previous case, we have $r\left(u \mid \Pi^{\prime}\right) \neq r\left(v \mid \Pi^{\prime}\right)$. Suppose thus that $u \in S_{i}$ and $v \in S_{j}$ with $i \neq j$. Notice that $d\left(u, S_{i}^{\prime}\right)=1$ and $N[v] \subseteq S_{j}$ because $v \in S_{j}$ and $v \in W^{\prime} \subseteq W$. Thus, $d\left(v, S_{i}\right) \geq 2$, and so $d\left(v, S_{i}^{\prime}\right)=d\left(v, S_{i}\right) \geq 2$. Finally, from $d\left(u, S_{i}^{\prime}\right) \neq d\left(v, S_{i}^{\prime}\right)$ we get that $r\left(u \mid \Pi^{\prime}\right) \neq r\left(v \mid \Pi^{\prime}\right)$.

The following result is a direct consequence of Theorem 1(2) and Theorem 10.

Corollary 11. If $G$ is a graph of order $n \geq 3$, then $\eta_{p}(G) \leq n-\operatorname{diam}(G)+2$. Moreover, this bound is sharp, and is attained, among others, by $P_{n}$ and $K_{1, n-1}$.

Proposition 12. If $G$ is a graph of order $n \geq 3$ and diameter $d$ such that $\eta_{p}(G)=k$, then $n \leq k\left(d^{k-1}-(d-1)^{k-1}\right)$.

Proof. Let $\Pi=\left\{S_{1}, \ldots, S_{k}\right\}$ be an RD-partition. If $u \in S_{i}$, then the $i$-th component of $r(u \mid \Pi)$ is 0 , any other component is a value from $\{1,2, \ldots, d\}$ and at least one component must be 1 . Since there are $d^{k-1}-(d-1)^{k-1}$ such $k$-tuples, we have that $\left|S_{i}\right| \leq d^{k-1}-(d-1)^{k-1}$, and therefore, $n \leq \sum_{i=1}^{k}\left|S_{i}\right| \leq k\left(d^{k-1}-(d-1)^{k-1}\right)$. 


\section{Extremal graphs}

In $[6,28]$, all graphs of order $n \geq 9$ satisfying $\beta_{p}(G)=n, \beta_{p}(G)=n-1$ and $\beta_{p}(G)=n-2$ were characterized. This section is devoted to approach the same problems for the dominating partition dimension $\eta_{p}(G)$. To this end, we prove a pair of technical lemmas.

Lemma 13. Let $k \geq 2$ be an integer. Let $G$ be a graph of order $n$ containing a vertex $u$ of degree d. If $n \geq 2 k+1$ and $k \leq d \leq n-k-1$, then $\eta_{p}(G) \leq n-k$.

Proof. Let $N(u)=\left\{x_{1}, \ldots, x_{k}, \ldots, x_{d}\right\}$. Let $L$ be the set containing all leaves at distance 2 from $u$ and let $C$ be the set containing both all non-leaves at distance 2 and all vertices at distance at least 3 from $u$, i.e., $C=V(G) \backslash(N[u] \cup L)$. Assume that $|L|=l$ and $|C|=c$ and observe that $l+c=n-d-1 \geq k$.

If $c \geq k$, then take the partition $\Pi=\left\{\left\{x_{1}, y_{1}\right\}, \ldots,\left\{x_{k}, y_{k}\right\}\right\} \cup\left\{\{z\}: z \notin\left\{x_{1}, \ldots, x_{k}, y_{1}, \ldots, y_{k}\right\}\right\}$, where $y_{1}, \ldots, y_{k} \in C$. Notice that $\Pi$ is a resolving partition since, for every $i \in\{1, \ldots, k\},\{u\}$ resolves the pair $x_{i}, y_{i}$, because $d\left(u, x_{i}\right)=1<2 \leq d\left(u, y_{i}\right)$. Furthermore, for every $i \in\{1, \ldots, k\}$, vertex $x_{i}$ is adjacent to $u$ and vertex $y_{i}$ is adjacent to a vertex different from $x_{i}$, because in the case $y_{i}$ has degree 1 , its neighbor does not belong to $N(u)$ by definition of $C$. So, $\Pi$ is also a dominating partition and thus $\eta_{p}(G) \leq n-k$.

Now, assume $c<k$. Let $h=k-c$ and observe that $1 \leq h \leq l$ since $l+c \geq k$. First, we seek if it is possible to pair $h$ vertices of $L$ with $h$ vertices of $N(u)$ satisfying that each pair is formed by non-adjacent vertices. Observe that this is equivalent to finding a matching $M$ that saturates a subset $L^{\prime}$ of $L$ of cardinality $h$ in the bipartite graph $H$ defined as follows: $N(u)$ and $L$ are its partite sets, and if $x_{i} \in N(u)$ and $z \in L$, then $x_{i} z \in E(H)$ if and only if $x_{i} z \notin E(G)$. So, the degree in $H$ of a vertex $z \in L$ is $\operatorname{deg}_{H}(z)=d-1$. For every nonempty set $W \subseteq L$ with $|W| \leq k-1$, we have $|W| \leq k-1 \leq d-1 \leq\left|N_{H}(W)\right|$, and for $W \subseteq L$ with $|W|=k$ we have $|W| \leq\left|N_{H}(W)\right|$ whenever $d \geq k+1$ or $\left|N_{H}(W)\right| \geq k$. Therefore, according to Hall's Theorem, there exists a matching $M$ saturating a subset $L^{\prime}$ of $L$ of cardinality $h$, except for the case $h=k=d$, provided that $\left|N_{H}(W)\right|<k$ for every subset $W \subseteq L$ with $|W|=k$. Let $M$ be such a matching, whenever it exists. We distinguish two cases.

Case 1: $h<k$. Consider the partition $\Pi$ formed by the $h$ pairs of the matching $M, c$ pairs formed by pairing the vertices in $C$ with $c$ vertices in $N(u)$ not used in the matching $M$, and a part for each one of the remaining vertices formed only by the vertex itself. Part $\{u\}$ resolves each part of cardinality 2 and, by construction, $\Pi$ is dominating. Thus, $\Pi$ is an RD-partition, implying that $\eta_{p}(G) \leq n-k$.

Case 2: $h=k$. In such a case, $c=0$ (i.e., $L=V(G) \backslash N[u]$ ). If $d>k$, then consider the partition $\Pi$ formed by the $k$ pairs of the matching $M$ and a part for each one of the remaining vertices formed only by the vertex itself. As in the preceding case, it can be shown that $\Pi$ is an RD-partition, and so $\eta_{p}(G) \leq n-k$.

If $d=h=k$ and there is a subset $W$ of $L$ of cardinality $k$ with $\left|N_{H}(W)\right| \geq k$, then there exists a matching $M$ between the vertices of $W$ and the vertices of $N(u)$. Consider the partition $\Pi$ formed by the $k$ pairs of the matching $M$ and a part for each one of the remaining vertices formed only by the vertex itself. As in the preceding case, it can be shown that $\Pi$ is an RD-partition, and so $\eta_{p}(G) \leq n-k$.

Finally, if $d=h=k$ and there is no subset $W$ of $L$ of cardinality $k$ with $\left|N_{H}(W)\right| \geq k$, then all vertices of $L$ are leaves hanging from the same vertex of $N(u)$. We may assume without loss of 
generality that all vertices in $L$ are adjacent to $x_{1}$. Let $y_{1}, \ldots, y_{k} \in L$ (they exist because $n \geq 2 k+1$ ). Consider the partition $\Pi=\left\{\left\{u, y_{1}\right\},\left\{x_{2}, y_{2}\right\}, \ldots,\left\{x_{k}, y_{k}\right\}\right\} \cup\left\{\{z\}: z \notin\left\{u, x_{2}, \ldots, x_{k}, y_{1}, \ldots, y_{k}\right\}\right\}$ (see Figure 2). Notice that $\Pi$ is a resolving partition since, for every $i \in\{2, \ldots, k\}, P_{1}=\left\{u, y_{1}\right\}$ resolves the pair $x_{i}, y_{i}$ because $d\left(x_{i}, P_{1}\right)=d\left(x_{i}, u\right)=1<2=d\left(y_{i}, P_{1}\right)$; and $P_{2}=\left\{x_{2}, y_{2}\right\}$ resolves the pair $u, y_{1}$, because $d\left(u, P_{2}\right)=d\left(u, x_{2}\right)=1<2=d\left(y_{1}, P_{2}\right)$. Besides, every vertex has a neighbor in another part by construction. Thus, $\Pi$ is an RD-partition, implying that $\eta_{p}(G) \leq n-k$.

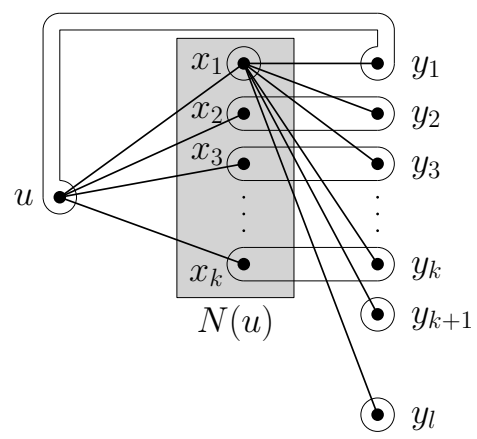

Figure 2: An RD-partition of cardinality $n-k$. There may be edges joining vertices of $N(u)$.

Lemma 14. Let $G$ be a graph order $n$.

(1) If $n \geq 5$ and $\operatorname{diam}(G) \geq 3$, then $\eta_{p}(G) \leq n-2$.

(2) If $n \geq 7$ and $\operatorname{diam}(G) \geq 4$, then $\eta_{p}(G) \leq n-3$.

Proof. (1) Let $\operatorname{diam}(G)=d$. If $d \geq 4$, then according to Corollary $11, \eta_{p}(G) \leq n-d+2 \leq n-2$. Assume thus that $d=3$ and take a vertex $u$ of eccentricity $\operatorname{ecc}(u)=3$. If $u$ is not a leaf, then $2 \leq \operatorname{deg}(u) \leq n-3$ and, by Lemma $13, \eta_{p}(G) \leq n-2$. If $u$ is a leaf, then consider the sets $D_{i}=\{v \mid d(u, v)=i\}, i \in\{1,2,3\}$. Take $x_{i} \in D_{i}, i \in\{1,2,3\}$ such that $\left\{u x_{1}, x_{1} x_{2}, x_{2} x_{3}\right\} \subseteq E(G)$. We distinguish cases depending on the cardinality of $D_{2}$.

Case 1: $\left|D_{2}\right| \geq 2$. Take a vertex $y_{2} \in D_{2}-x_{2}$. Note that $x_{1} y_{2} \in E(G)$, since $u$ is a leaf. Take the partition:

$$
\Pi=\left\{\left\{x_{1}, x_{2}\right\},\left\{x_{3}, y_{2}\right\} \cup\left\{\{z\}: z \neq x_{1}, x_{2}, x_{3}, y_{2}\right\} .\right.
$$

Clearly, $\Pi$ is an RD-partition of $G$ of cardinality $n-2$. Thus, $\eta_{p}(G) \leq n-2$.

Case 2: $\left|D_{2}\right|=1$. Notice that $\left|D_{3}\right| \geq 2$ since $n \geq 5$. Take a vertex $y_{3} \in D_{3}-x_{3}$. Observe that $x_{2} y_{3} \in E(G)$. Take the partition:

$$
\Pi=\left\{\left\{x_{1}, x_{2}\right\},\left\{u, y_{3}\right\} \cup\left\{\{z\}: z \neq u, x_{1}, x_{2}, y_{3}\right\} .\right.
$$

Clearly, $\Pi$ is an RD-partition of $G$ of cardinality $n-2$. Thus, $\eta_{p}(G) \leq n-2$.

(2) If $d \geq 5$, then according to Corollary $11, \eta_{p}(G) \leq n-d+2 \leq n-3$. Assume thus that $d=4$ and take a vertex $u$ of eccentricity of $\operatorname{ecc}(u)=4$. Notice that $\operatorname{deg}(u) \leq n-4$ and hence, according to Lemma 13 (case $k=3$ ), $\eta_{p}(G) \leq n-3$ whenever $\operatorname{deg}(u) \geq 3$. Suppose finally that $1 \leq \operatorname{deg}(u) \leq 2$ and consider the sets $D_{i}=\{v \mid d(u, v)=i\}, i \in\{1,2,3,4\}$. Notice that $1 \leq\left|D_{1}\right| \leq 2$. Take $x_{i} \in D_{i}$, $i \in\{1,2,3,4\}$ such that $\left\{u x_{1}, x_{1} x_{2}, x_{2} x_{3}, x_{3} x_{4}\right\} \subseteq E(G)$. We distinguish cases depending on the cardinality of $D_{1}$ and $D_{2}$. 
Case 1: $\left|D_{1}\right|=2$. Take a vertex $y_{1} \in D_{1}-x_{1}$. Take the partition:

$$
\Pi=\left\{\left\{u, x_{1}\right\},\left\{x_{2}, x_{3}\right\},\left\{x_{4}, y_{1}\right\} \cup\left\{\{z\}: z \neq u, x_{1}, x_{2}, x_{3}, x_{4}, y_{1}\right\} .\right.
$$

Clearly, $\Pi$ is an RD-partition of $G$ of cardinality $n-3$. Thus, $\eta_{p}(G) \leq n-3$.

Case 2: $\left|D_{1}\right|=1$ and $\left|D_{2}\right| \geq 2$. Take a vertex $y_{2} \in D_{2}-x_{2}$. Take the partition:

$$
\Pi=\left\{\left\{u, x_{4}\right\},\left\{x_{1}, x_{2}\right\},\left\{x_{3}, y_{2}\right\} \cup\left\{\{z\}: z \neq u, x_{1}, x_{2}, x_{3}, x_{4}, y_{2}\right\} .\right.
$$

Clearly, $\Pi$ is an RD-partition of $G$ of cardinality $n-3$. Thus, $\eta_{p}(G) \leq n-3$.

Case 3: $\left|D_{1}\right|=1,\left|D_{2}\right|=1$ and $\left|D_{3}\right| \geq 2$. Take a pair of vertices $y_{3}, w \in D_{3} \cup D_{4} \backslash\left\{x_{3}, x_{4}\right\}$ such that $y_{3} \in D_{3}$. Take the partition:

$$
\Pi=\left\{\left\{x_{1}, w\right\},\left\{x_{2}, x_{3}\right\},\left\{x_{4}, y_{3}\right\} \cup\left\{\{z\}: z \neq x_{1}, x_{2}, x_{3}, x_{4}, y_{3}, w\right\} .\right.
$$

Clearly, $\Pi$ is an RD-partition of $G$ of cardinality $n-3$. Thus, $\eta_{p}(G) \leq n-3$.

Case 4: $\left|D_{1}\right|=1,\left|D_{2}\right|=1$ and $\left|D_{3}\right|=1$. Take a pair of vertices $y_{4}, w_{4} \in D_{4}-x_{4}$. Note that $\left\{x_{3} y_{4}, x_{3} w_{4}\right\} \subseteq E(G)$. Take the partition:

$$
\Pi=\left\{\left\{u, y_{4}\right\},\left\{x_{1} w_{4}\right\},\left\{x_{2}, x_{3}\right\} \cup\left\{\{z\}: z \neq u, x_{1}, x_{2}, x_{3}, x_{4}, y_{4}, w_{4}\right\} .\right.
$$

Clearly, $\Pi$ is an RD-partition of $G$ of cardinality $n-3$. Thus, $\eta_{p}(G) \leq n-3$.

In [6], all graphs of order $n$ satisfying $n-1 \leq \beta_{p} \leq n$ were characterized (see Theorem 1). We display a similar result for the dominating partition dimension $\eta_{p}$.

Theorem 15. If $G$ is a graph of order $n \geq 6$, then

(1) $\eta_{p}(G)=n$ if and only if $G$ is isomorphic to either the complete graph $K_{n}$ or the star $K_{1, n-1}$.

(2) $\eta_{p}(G)=n-1$ if and only if $G$ is isomorphic to either the complete split graph $K_{n-2} \vee \overline{K_{2}}$, or the graph $K_{1} \vee\left(K_{1}+K_{n-2}\right)$.

Proof. (1) According to Theorem 10, if $\eta_{p}(G)=n$ then $n-1 \leq \beta_{p}(G) \leq n$. By direct inspection on graphs with $\beta_{p}(G)=n$ and $\beta_{p}(G)=n-1$ (see Theorem 1) the stated result is derived.

(2) It is a routine exercise to check that $\eta_{p}\left(K_{n-2} \vee \overline{K_{2}}\right)=\eta_{p}\left(K_{1} \vee\left(K_{1}+K_{n-2}\right)\right)=n-1$. Conversely, let $G$ be a graph such that $\eta_{p}(G)=n-1$. By Lemma $14(1)$, $\operatorname{diam}(G)=2$, since $G \neq K_{n}$. Take a pair of vertices $u, v$ such that $d(u, v)=2$. By Lemma 13 (case $k=2$ ), $\operatorname{deg}(u), \operatorname{deg}(v) \in\{1, n-2\}$. We distinguish three cases.

Case 1: $\operatorname{deg}(u)=\operatorname{deg}(v)=1$. Let $w$ be the vertex such that $N(u)=N(v)=\{w\}$. By Lemma 13, the rest of vertices of $G$ have degree 1, as they are not adjacent neither to $u$ nor to $v$. Hence, all vertices of $G$ other than vertex $w$ are leaves hanging from $w$, i.e., $G \cong K_{1, n-1}$, a contradiction.

Case 2: $\operatorname{deg}(u)=\operatorname{deg}(v)=n-2$. In this case, $N(u)=N(v)=V(G) \backslash\{u, v\}=W$ and for all vertex $z \in W, \operatorname{deg}(z) \geq 2$. Then, by Lemma 13 (case $k=2$ ), $\operatorname{deg}(z) \in\{n-2, n-1\}$.

If $\operatorname{deg}(z)=n-1$ for all $z \in W$, then $G$ is isomorphic to the complete split graph $K_{n-2} \vee \overline{K_{2}}$. 
If there is a vertex $t \in W$ such that $\operatorname{deg}(t)=n-2$, then let $s \in W$ be the vertex that is not adjacent to $t$. Observe that both $t$ and $s$ are adjacent to any other vertex of $W$. If $a, b \in W \backslash\{s, t\}$, then $\Pi=\{\{u, a\},\{s, b\}\} \cup\{\{z\}: z \neq a, b, u, s\}$ is an RD-partition, and thus $\eta_{p}(G) \leq n-2$.

Case 3: $\operatorname{deg}(u)=1$ and $\operatorname{deg}(u)=n-2$. Let $w$ be the vertex adjacent to $u$. Since the diameter is 2 , every vertex $t \notin\{u, w, v\}$ is adjacent both to $w$ and $v$. In particular, for all vertex $t \notin\{u, w, v\}, \operatorname{deg}(t) \geq 2$ and, by Lemma 13 (case $k=2$ ), $\operatorname{deg}(t)=n-2$ and then $G$ is isomorphic to the graph $K_{1} \vee\left(K_{1}+K_{n-2}\right)$.

Next, we characterize those graphs with $\eta_{p}(G)=n-2$. Concretely, we prove that, for every integer $n \geq 7$, a graph of order $n$ satisfies $\eta_{p}(G)=n-2$ if and only if it belongs to the family $\Lambda_{n}=\left\{H_{1}, \ldots, H_{17}\right\}$ (see Figure 3 ).
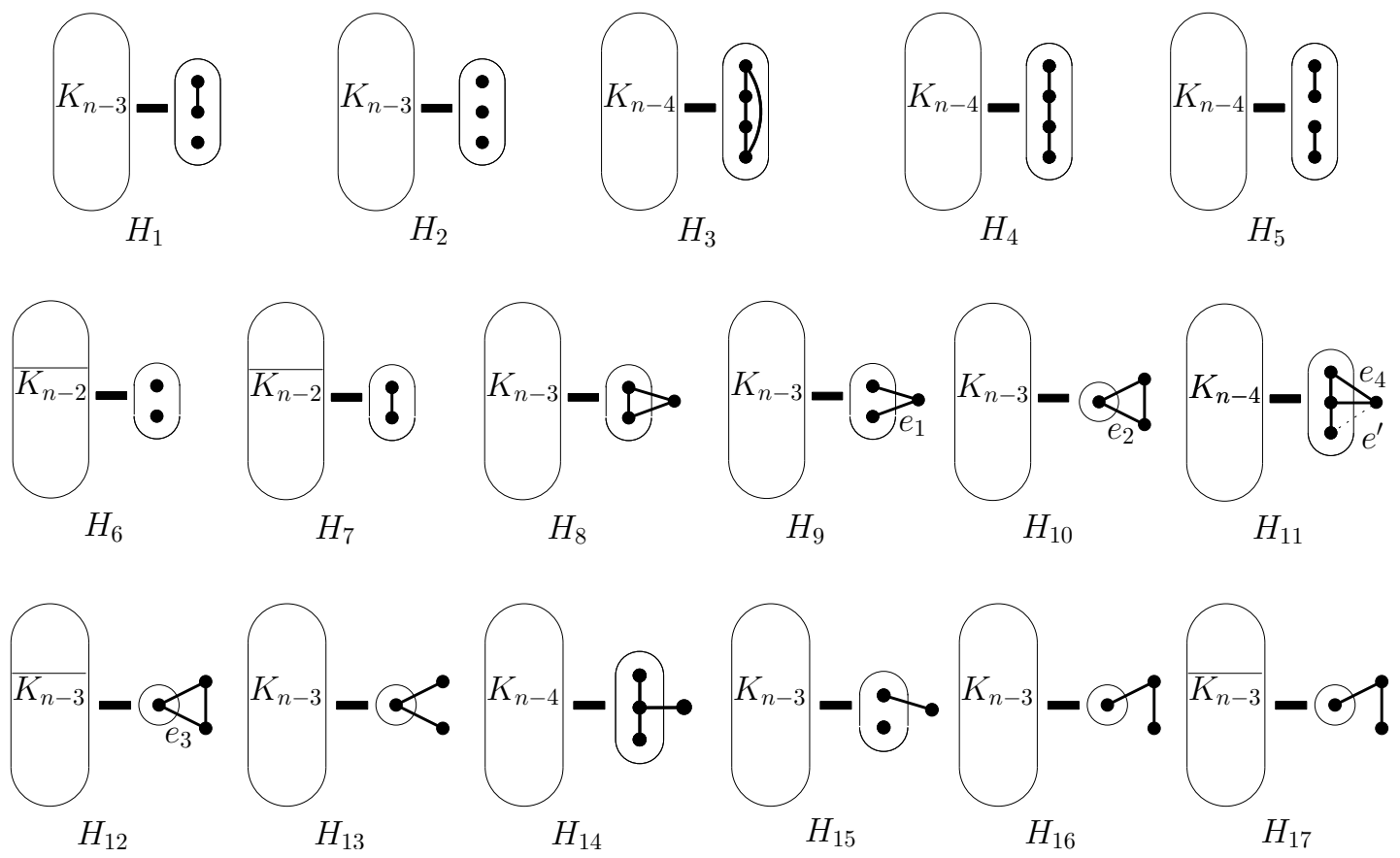

$$
\begin{array}{lll}
H_{1} \cong K_{n-3} \vee\left(K_{2}+K_{1}\right) & H_{2} \cong K_{n-3} \vee \overline{K_{3}} & H_{3} \cong K_{n-4} \vee C_{4} \\
H_{4} \cong K_{n-4} \vee P_{4} & H_{5} \cong K_{n-4} \vee 2 K_{2} & H_{6} \cong K_{2, n-2} \\
H_{7} \cong \overline{K_{n-2}} \vee K_{2} & H_{8} \cong\left(K_{n-3}+K_{1}\right) \vee K_{2} & H_{9} \cong\left(K_{n-3}+K_{1}\right) \vee \overline{K_{2}} \\
H_{10} \cong\left(K_{n-3}+K_{2}\right) \vee K_{1} & H_{11} \cong\left(K_{n-4}+K_{1}\right) \vee P_{3}-e^{\prime} & H_{12} \cong\left(\overline{K_{n-3}}+K_{2}\right) \vee K_{1} \\
H_{13} \cong\left(K_{n-3}+\overline{K_{2}}\right) \vee K_{1} & H_{14} \cong H_{11}-e_{4} & H_{15} \cong H_{9}-e_{1} \\
H_{16} \cong H_{10}-e_{2} & H_{17} \cong H_{12}-e_{3} &
\end{array}
$$

Figure 3: The family $\Lambda_{n}$ of all graphs of order $n \geq 7$ such that $\eta_{p}(G)=n-2$. 
Proposition 16. If $G \in \Lambda_{n}=\left\{H_{1}, \ldots, H_{17}\right\}$, then $\eta_{p}(G)=n-2$. Moreover, if $G \in \Lambda_{n} \backslash\left\{H_{12}, H_{17}\right\}$, then $\beta_{p}(G)=n-2$.

Proof. According to Theorem 15, for every graph $H_{i} \in \Lambda_{n}, \beta_{p}(G) \leq \eta_{p}(G) \leq n-2$. Thus, it is enough to check that, for every graph $H_{i} \in \Lambda_{n}, \eta_{p}\left(H_{i}\right) \geq n-2$, and also that if $i \notin\{12,17\}$, then $\beta_{p}\left(H_{i}\right) \geq n-2$.

Case 1: If $G \in\left\{H_{6}, H_{7}\right\}$, then it contains a twin set $W$ of cardinality $n-2$ (see Figure 3 ) and thus, by Lemma $8, \eta_{p}(G) \geq \beta_{p}(G) \geq n-2$.

Case 2: If $G \in\left\{H_{1}, H_{2}, H_{8}, H_{9}, H_{10}, H_{13}, H_{15}, H_{16}\right\}$, then there exists a set of vertices $W$ of $n-3$ vertices of $G$ such that $W$ induces a complete graph (see Figure 3), and thus, according to Lemma $8, \eta_{p}(G) \geq \beta_{p}(G) \geq(n-3)+1=n-2$.

Case 3: If $G \in\left\{H_{12}, H_{17}\right\}$, then $G$ is a graph with a twin set of $n-3$ leaves (see Figure 3 ) and, by Lemma $8, \beta_{p}(G) \geq n-3$ and $\eta_{p}(G) \geq(n-3)+1=n-2$.

Case 4: If $G \in\left\{H_{3}, H_{4}, H_{5}, H_{11}, H_{14}\right\}$, then there exists a twin set $W$ of cardinality $n-4$ that $W$ induces a complete graph (see Figure 3), and thus, by Lemma 8, $\eta_{p}(G) \geq \beta_{p}(G) \geq(n-4)+1=n-3$. Suppose that there exists a resolving partition $\Pi=\left\{S_{1}, \ldots, S_{n-3}\right\}$ of cardinality $n-3$. Assume that $W=\left\{w_{1}, \ldots, w_{n-4}\right\}$ and $w_{i} \in S_{i}$, for every $i \in\{1, \ldots, n-4\}$, so that $S_{n-3} \cap W=\emptyset$. Notice also that all these graphs have diameter 2 . We distinguish two cases.

Case 4.1: If $G \in\left\{H_{3}, H_{4}, H_{5}\right\}$, then $N[W]=V(G)$ and $|V(G) \backslash W|=4$. Clearly, $\left|S_{n-3}\right|=1$, since $r(z \mid \Pi)=(1, \ldots, 1,0)$ for every $z \in S_{n-3}$. Notice also that $\left|S_{i}\right| \leq 2$ for $i \in\{1, \ldots, n-4\}$, since for every $x \in S_{i}$ we have $r(x \mid \Pi)=(1, \ldots, 1, \stackrel{i)}{0}, 1, \ldots, 1, h)$, with $h \in\{1,2\}$. Hence, there must be exactly three sets of $\Pi$ of cardinality 2 and we can suppose without loss of generality that $S_{1}=\left\{w_{1}, x\right\}, S_{2}=\left\{w_{2}, y\right\}, S_{3}=\left\{w_{3}, z\right\}$ and $S_{n-3}=\{t\}$, where $\{x, y, z, t\}=V(G) \backslash W$. We know that $d\left(t, w_{1}\right)=d\left(t, w_{2}\right)=d\left(t, w_{3}\right)=1$, hence $d(t, x)=d(t, y)=d(t, z)=2$, a contradiction, because there is no vertex satisfying this condition in $V(G) \backslash W$.

Case 4.2: If $G \in\left\{H_{11}, H_{14}\right\}$, then $|N[W] \backslash W|=3$. We may assume $N[W] \backslash W=\{a, b, c\}$ and $V(G) \backslash N[W]=\{z\}$ with $d(a, b)=d(b, c)=1, d(b, z)=1$ and $d(c, z)=2$ in both graphs. Notice that $S_{n-3}$ has as most one vertex from $\{a, b, c\}$, since $r(x \mid \Pi)=(1, \ldots, 1,0)$ whenever $x \in\{a, b, c\} \cap S_{n-3}$. Moreover, $b \notin S_{n-3}$, because if $b \in S_{n-3}$, then $a \notin S_{n-3}$ so that $a \in S_{i}$, for some $i \in\{1, \ldots, n-4\}$, i)

and then $r(a \mid \Pi)=r\left(w_{i} \mid \Pi\right)=(1, \ldots, 1,0,1, \ldots, 1,1)$, a contradiction. So, we can assume without loss of generality that $\left\{w_{1}, b\right\} \subseteq S_{1}$. Thus, $S_{n-3}=\{z\}$, otherwise $a$ or $c$ should belong to $S_{n-3}$, so that $r\left(w_{1} \mid \Pi\right)=r(b \mid \Pi)=(0,1, \ldots, 1,1)$, a contradiction. Hence $c \in S_{j}$, for some $j \in\{1, \ldots, n-4\}$, but then $r\left(w_{j} \mid \Pi\right)=r(c \mid \Pi)=(1, \ldots, 1, \stackrel{j}{0}, 1 \ldots, 1,2)$, a contradiction.

The remainder of this section is devoted to showing that these 17 graph families are the only ones satisfying $\eta_{p}(G)=n-2$.

First, note that as a direct consequence of Lemma 14(2) the following result is derived.

Corollary 17. If $G$ is a graph with $\eta_{p}(G)=n-2$, then $2 \leq \operatorname{diam}(G) \leq 3$. 


\subsection{Case diameter 2}

Let $G$ be a graph such that $\eta_{p}(G)=n-2$ and $\operatorname{diam}(G)=2$. We distinguish two cases depending whether $\delta(G) \geq n-3$ or $\delta(G) \leq n-4$. To approach the first case (notice that the restriction $\operatorname{diam}(G)=2$ is redundant) we need the following technical lemma.

Lemma 18. Let $G$ be a graph of order $n \geq 7$ and minimum degree $\delta(G)$ at least $n-3$. If $G$ contains at most $n-5$ vertices of degree $n-1$, then $\eta_{p}(G) \leq n-3$.

Proof. Observe that the complement $\bar{G}$ of $G$ is a (non-necessarily connected) graph with vertices of degree 0,1 or 2 . Thus, the components of $\bar{G}$ are either isolated vertices, or paths of order at least 2 , or cycles of order at least 3 . By hypothesis, $G$ has at most $n-5$ vertices of degree $n-1$, therefore $\bar{G}$ has at least 5 vertices of degree 1 or 2 . We distinguish three cases.

Case 1: $\bar{G}$ has only one non-trivial component. In such a case, $\bar{G}$ has al least a (non-necessarily induced) subgraph isomorphic to $P_{5}$. Let $x_{1}, x_{2}, x_{3}, x_{4}$ and $x_{5}$ be the vertices of this path, where $x_{i} x_{i+1} \in E(\bar{G})$ for $i=1,2,3,4$. Let $z \notin\left\{x_{1}, x_{2}, x_{3}, x_{4}, x_{5}\right\}$. Consider the partition:

$$
\Pi=\left\{\left\{x_{1}, x_{3}, x_{5}, z\right\}\right\} \cup\left\{\{v\}: v \notin\left\{x_{1}, x_{3}, x_{5}, z\right\}\right\} .
$$

We claim that $\Pi$ is an RD-partition of $G$ (see Figure 4 (a)). Indeed, if $S_{1}=\left\{x_{2}\right\}$ and $S_{2}=\left\{x_{4}\right\}$, then $r\left(x_{1} \mid \Pi\right)=(2,1, \ldots), r\left(x_{3} \mid \Pi\right)=(2,2, \ldots), r\left(x_{5} \mid \Pi\right)=(1,2, \ldots), r(z \mid \Pi)=(1,1, \ldots)$. Moreover, $x_{3}$ is adjacent in $G$ to any vertex $w \notin\left\{x_{1}, x_{2}, x_{3}, x_{4}, x_{5}, z\right\}$, that exists because the order of $G$ is at least 7 . Therefore, $\Pi$ is an RD-partition of $G$. Thus, $\eta_{p}(G) \leq n-3$.

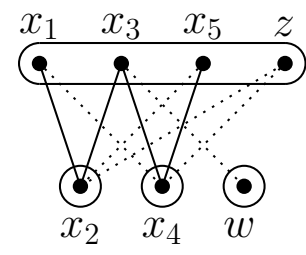

(a)

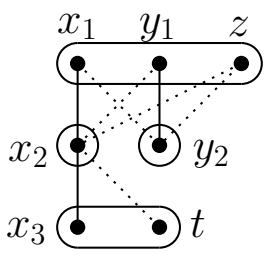

$(b)$

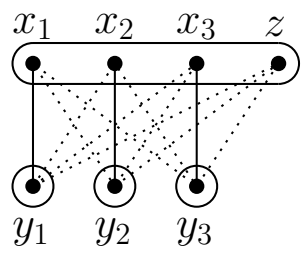

$(c)$

Figure 4: Solid (resp. dotted) lines mean adjacent (resp. non-adjacent) vertices in $\bar{G}$.

Case 2: $\bar{G}$ has at least two non-trivial components and one of them has order at least 3. If there is only one component of order $\geq 3$, say $C_{1}$, then there is at least a component of order 2 , say $C_{2}$. Otherwise, there are two components, say $C_{1}$ and $C_{2}$, of order at least 3 . In both cases, we may assume that $x_{1}, x_{2}, x_{3}$ are vertices of $C_{1}$ and $y_{1}, y_{2}$ are vertices of $C_{2}$, such that $x_{1} x_{2} \in E(\bar{G})$, $x_{2} x_{3} \in E(\bar{G}), y_{1} y_{2} \in E(\bar{G})$. Since $n \geq 7$, we may assume that there are two more vertices $z$ and $t$ such that at least one of them, say $z$, is not adjacent to $y_{2}$ in $\bar{G}$.

Consider the partition:

$$
\Pi=\left\{\left\{x_{1}, y_{1}, z\right\},\left\{x_{3}, t\right\}\right\} \cup\left\{\{v\}: v \notin\left\{x_{1}, x_{3}, y_{1}, t, z\right\}\right\} .
$$

We claim that $\Pi$ is an RD-partition of $G$ (see Figure 4 (b)). Indeed, recall that two vertices are at distance 2 in $G$ whenever they are adjacent in $\bar{G}$, and they are at distance 1 in $G$ whenever 


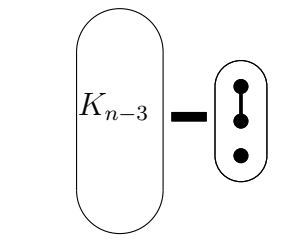

$H_{1} \cong K_{n-3} \vee\left(K_{2}+K_{1}\right)$

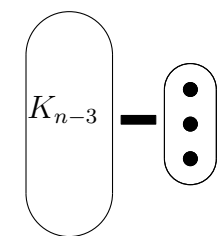

$H_{2} \cong K_{n-3} \vee \overline{K_{3}}$

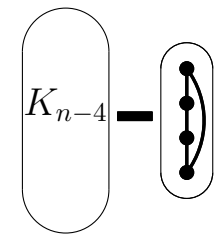

$H_{3} \cong K_{n-4} \vee C_{4}$

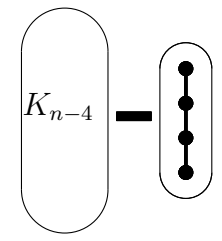

$H_{4} \cong K_{n-4} \vee P_{4}$

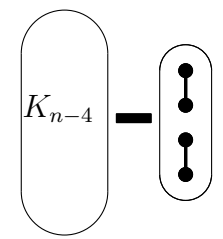

$H_{5} \cong K_{n-4} \vee 2 K_{2}$

Figure 5: Graphs of order $n \geq 7$, diameter $\operatorname{diam}(G)=2$ and minimum degree $\delta(G) \geq n-3$ such that $\eta(G)=n-2$.

they are not adjacent in $\bar{G}$. Hence, if $S_{1}=\left\{x_{2}\right\}$ and $S_{2}=\left\{y_{2}\right\}$, then $r\left(x_{1} \mid \Pi\right)=(2,1, \ldots)$, $r\left(y_{1} \mid \Pi\right)=(1,2, \ldots), r(z \mid \Pi)=(1,1, \ldots)$, and $r\left(x_{3} \mid \Pi\right)=(2, \ldots), r(t \mid \Pi)=(1, \ldots)$. Therefore, $\Pi$ is an RD-partition of $G$ and $\eta_{p}(G) \leq n-3$.

Case 3: All non-trivial components of $\bar{G}$ have order 2. Then, $\bar{G}$ has at least 3 components that are copies of $K_{2}$. Let $\left\{x_{i}, y_{i}\right\}$, for $i=1,2,3$, be the vertices of three of these copies, and let $z$ be a vertex not belonging to them. Then,

$$
\Pi=\left\{\left\{x_{1}, x_{2}, x_{3}, z\right\}\right\} \cup\left\{\{v\}: v \neq x_{1}, x_{2}, x_{3}, z\right\}
$$

is an RD-partition of $G$ (see Figure 4 (c)). Indeed, if $S_{1}=\left\{y_{1}\right\}, S_{2}=\left\{y_{2}\right\}$ and $S_{3}=\left\{y_{3}\right\}$, then $r\left(x_{1} \mid \Pi\right)=(2,1,1, \ldots), r\left(x_{2} \mid \Pi\right)=(1,2,1, \ldots), r\left(x_{3} \mid \Pi\right)=(1,1,2, \ldots)$ and $r(z \mid \Pi)=(1,1,1, \ldots)$. Therefore, $\eta_{p}(G) \leq n-3$.

Proposition 19. Let $G$ be a graph of order $n \geq 7$, diameter 2 and minimum degree at least $n-3$. If $\eta_{p}(G)=n-2$, then $G \in\left\{H_{1}, H_{2}, H_{3}, H_{4}, H_{5}\right\}$ (see Figure 5).

Proof. Let $\Omega \subseteq V(G)$ be the set of vertices of $G$ of degree $n-1$, which according to Lemma 18 contains at least $n-4$ vertices. We distinguish cases depending on the cardinality of $\Omega$.

Case 1: $|\Omega| \geq n-2$. If $|\Omega|=n$, then $G \cong K_{n}$ and thus $\eta_{p}(G)=n$. Case $|\Omega|=n-1$ is not possible. If $|\Omega|=n-2$, then $G \cong K_{n-2} \vee \overline{K_{2}}$, and according to Theorem $15(2), \eta_{p}(G)=n-1$.

Case 2: $|\Omega|=n-3$. Let $F$ be the subgraph of order 3 induced by $V(G) \backslash \Omega$, i.e., $F=G[V(G) \backslash \Omega]$. Notice that $|E(F)| \leq 1$. If $|E(F)|=1$, then $G \cong H_{1}$. Otherwise, if $|E(F)|=0$, then $G \cong H_{2}$.

Case 3: $|\Omega|=n-4$. Consider the graph of order $4, F=G[V(G) \backslash \Omega]$. Note that all vertices of $F$ have degree either 1 or 2 . There are thus three possibilities. If $F \cong C_{4}$, then $G \cong H_{3}$. If $F \cong P_{4}$, then $G \cong H_{4}$. If $F \cong 2 K_{2}$, then $G \cong H_{5}$.

Proposition 20. Let $G$ be a graph of order $n \geq 7$, diameter 2 and minimum degree at most $n-4$. If $\eta_{p}(G)=n-2$, then $G \in\left\{H_{6}, H_{7}, H_{8}, H_{9}, H_{10}, H_{11}, H_{12}, H_{13}, H_{14}\right\}$ (see Figure 6 ).

Proof. By Lemma 13 for $k=3$, we have that $\operatorname{deg}(w) \in\{1,2, n-3, n-2, n-1\}$, for every vertex $w \in V(G)$. Hence, $\delta(G) \leq 2$. We distinguish two cases.

Case 1: There exists a vertex $u$ of degree 2. Consider the subsets $D_{1}=N(u)=\left\{x_{1}, x_{2}\right\}$ and $D_{2}=\{v \in V(G): d(u, v)=2\}=V(G) \backslash N[u]$, so that $\left|D_{2}\right|=n-3$.

Case 1.1: $G\left[D_{2}\right]$ is neither complete nor empty. Then, there exist three different vertices $r, s, t \in D_{2}$ such that $r s \in E(G)$ and $r t \notin E(G)$. Let $y \in D_{2} \backslash\{r, s, t\}$. We distinguish cases taking into account whether or not $y$ and $t$ are leaves. 
- Both $y$ and $t$ are leaves hanging from the same vertex. Assume that they hang from $x_{1}$. Let $S_{1}=\{u, y\}$ and $S_{2}=\left\{x_{2}, s, t\right\}$. In such a case, $S_{2}$ resolves $S_{1},\{r\}$ resolves the pair $\{s, t\}$ and $S_{1}$ resolves the pairs $\left\{x_{2}, s\right\}$ and $\left\{x_{2}, t\right\}$. Therefore, $\Pi=\left\{S_{1}, S_{2}\right\} \cup\left\{\{w\}: w \notin S_{1} \cup S_{2}\right\}$ is a resolving partition. It can be easily checked that $\Pi$ is also dominating. Hence, $\eta_{p}(G) \leq n-3$, a contradiction.

- Both y and $t$ are leaves but not hanging from the same vertex, or neither $y$ nor $t$ are leaves. If both $y$ and $t$ are leaves but not hanging from the same vertex, assume $x_{1} y \in E$ and $x_{2} t \in E$. Let $S_{1}=\left\{x_{2}, y\right\}$ and $S_{2}=\left\{x_{1}, s, t\right\}$. If neither $y$ nor $t$ are leaves and $N(t) \neq\left\{s, x_{1}\right\}$, let $S_{1}=\left\{x_{2}, y\right\}$ and $S_{2}=\left\{x_{1}, s, t\right\}$. If neither $y$ nor $t$ are leaves and $N(t)=\left\{s, x_{1}\right\}$, let $S_{1}=\left\{x_{1}, y\right\}$ and $S_{2}=\left\{x_{2}, s, t\right\}$. In all these cases, $\{u\}$ resolves $S_{1},\{r\}$ resolves $\{s, t\}$, and $\{u\}$ resolves any other pair from $S_{2}$. Hence, $\Pi=\left\{S_{1}, S_{2}\right\} \cup\left\{\{w\}: w \notin S_{1} \cup S_{2}\right\}$ is a resolving partition of $G$. It can be easily checked that $\Pi$ is a dominating partition. Thus, $\eta_{p}(G) \leq n-3$, a contradiction.

- Exactly one of the vertices $y$ or $t$ is a leaf. We may assume that the leaf hangs from $x_{1}$. If $t$ is a leaf, then take $S_{1}=\left\{x_{1}, y\right\}$ and $S_{2}=\left\{x_{2}, s, t\right\}$. If $y$ is a leaf and $N(t) \neq\left\{x_{1}, s\right\}$ then take $S_{1}=\left\{x_{2}, y\right\}$ and $S_{2}=\left\{x_{1}, s, t\right\}$. In both cases, $\{r\}$ resolves $\{s, t\}$ and $\{u\}$ resolves any other pair in either $S_{1}$ or $S_{2}$. If $y$ is a leaf and $N(t)=\left\{x_{1}, s\right\}$ then take $S_{1}=\{u, y\}$ and $S_{2}=\left\{x_{2}, s, t\right\}$. Then, $\{r\}$ resolves the pair $\{s, t\}, S_{1}$ resolves the other pairs from $S_{2} ;$ and $S_{2}$ resolves $S_{1}$. In all cases, $\Pi=\left\{S_{1}, S_{2}\right\} \cup\left\{\{w\}: w \notin S_{1} \cup S_{2}\right\}$ is dominating partition. Thus, $\eta_{p}(G) \leq n-3$, a contradiction.

Case 1.2: $G\left[D_{2}\right]$ is either complete or empty. Assume that $\operatorname{deg}\left(x_{1}\right) \leq \operatorname{deg}\left(x_{2}\right)$. Consider the subsets $N_{1}=N\left(x_{1}\right) \cap D_{2}$ and $N_{2}=N\left(x_{2}\right) \cap D_{2}$. Observe that $N_{1} \cup N_{2}=D_{2}$, and the sets $N_{1} \backslash N_{2}, N_{1} \cap N_{2}$ and $N_{2} \backslash N_{1}$ are pairwise disjoint. Besides, $\left|N_{2} \backslash N_{1}\right| \geq\left|N_{1} \backslash N_{2}\right|$ because we have assumed $\operatorname{deg}\left(x_{2}\right) \geq \operatorname{deg}\left(x_{1}\right)$. Notice also that $\operatorname{deg}\left(x_{2}\right) \geq \operatorname{deg}\left(x_{1}\right) \geq 2$, as otherwise $\operatorname{diam}(G) \geq 3$. We distinguish two cases.

(1.2.1): $\operatorname{deg}\left(x_{1}\right)=2$. Thus, $\operatorname{deg}\left(x_{2}\right) \geq\left(\left|D_{2}\right|-1\right)+1 \geq n-3$.

- If $x_{1} x_{2} \in E$, then $N_{1}=\emptyset$ and $D_{2}=N_{2}$. If $G\left[D_{2}\right] \cong K_{n-3}$, then $G \cong H_{10}$. If $G\left[D_{2}\right] \cong \overline{K_{n-3}}$, then $G \cong H_{12}$.

- If $x_{1} x_{2} \notin E$, then $\left|N_{1}\right|=1$ and $\left|N_{2} \backslash N_{1}\right|=n-4 \geq 3$. If $G\left[D_{2}\right] \cong \overline{K_{n-3}}$, then $\operatorname{diam}(G) \geq 3$. Hence, $G\left[D_{2}\right] \cong K_{n-3}$. Consider $y \in N_{1}$ and $z_{1}, z_{2} \in N_{2} \backslash N_{1}$. Let $S_{1}=\left\{u, x_{1}\right\}, S_{2}=\left\{x_{2}, z_{2}\right\}$ and $S_{3}=\left\{y, z_{1}\right\}$ and consider the partition $\Pi=\left\{S_{1}, S_{2}, S_{3}\right\} \cup\left\{\{w\}: w \notin S_{1} \cup S_{2} \cup S_{3}\right\}$. Then, $S_{1}$ resolves both $S_{2}$ and $S_{3}$; and $S_{3}$ resolves $S_{1}$. Moreover, $\Pi$ is a dominating partition of $G$. Thus, $\eta_{p}(G) \leq n-3$, a contradiction.

(1.2.2): $\operatorname{deg}\left(x_{1}\right) \geq n-3$. Hence, $\operatorname{deg}\left(x_{2}\right) \geq \operatorname{deg}\left(x_{1}\right) \geq n-3$. In such a case, $\left|N_{1}\right| \geq n-5$ and $\left|N_{2}\right| \geq n-5$, and so $n-7 \leq\left|N_{1} \cap N_{2}\right| \leq n-3$. We distinguish cases depending on the cardinality of $\left|N_{1} \cap N_{2}\right|$.

- $\left|N_{1} \cap N_{2}\right|=n-3$. Then, $N_{1}=N_{2}=V(G) \backslash N[u]$. If $x_{1} x_{2} \in E$, then $G \cong H_{8}$ if $G\left[D_{2}\right] \cong K_{n-3}$, and $G \cong H_{7}$ if $G\left[D_{2}\right] \cong \overline{K_{n-3}}$. If $x_{1} x_{2} \notin E$, then $G \cong H_{9}$ if $G\left[D_{2}\right] \cong K_{n-3}$, and $G \cong H_{6}$ if $G\left[D_{2}\right] \cong \overline{K_{n-3}}$. 


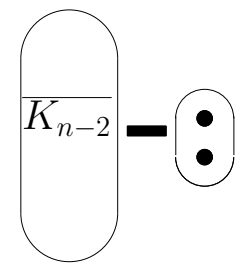

$H_{6} \cong K_{2, n-2}$

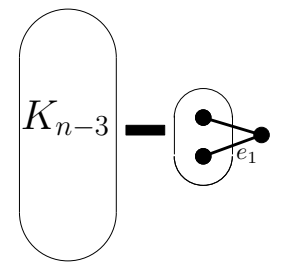

$H_{9} \cong\left(K_{n-3}+K_{1}\right) \vee \overline{K_{2}}$

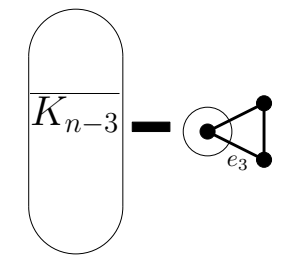

$H_{12} \cong\left(\overline{K_{n-3}}+K_{2}\right) \vee K_{1}$

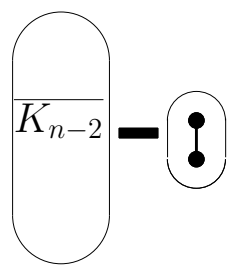

$H_{7} \cong \overline{K_{n-2}} \vee K_{2}$

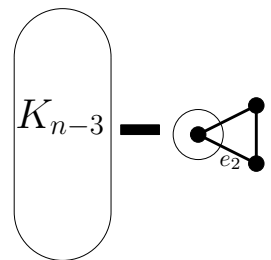

$H_{10} \cong\left(K_{n-3}+K_{2}\right) \vee K_{1}$

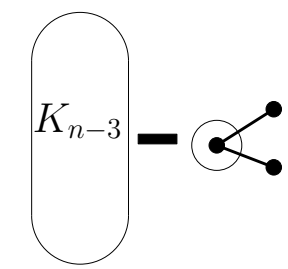

$H_{13} \cong\left(K_{n-3}+\overline{K_{2}}\right) \vee K_{1}$

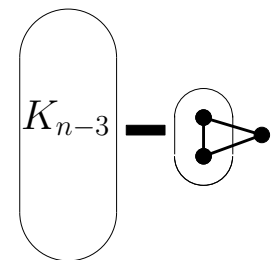

$H_{8} \cong\left(K_{n-3}+K_{1}\right) \vee K_{2}$

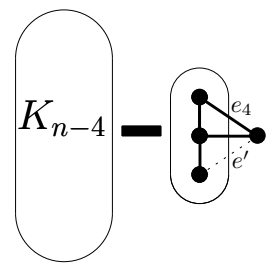

$H_{11} \cong\left(K_{n-4}+K_{1}\right) \vee P_{3}-e^{\prime}$

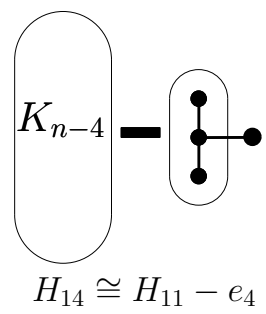

Figure 6: Graphs of order $n \geq 7$, diameter $\operatorname{diam}(G)=2$ and minimum degree $1 \leq \delta(G) \leq 2$ such that $\eta(G)=n-2$.

- $\left|N_{1} \cap N_{2}\right|=n$ - 4. Then, $\left|N_{2} \backslash N_{1}\right|+\left|N_{1} \backslash N_{2}\right|=1$. Thus, $\left|N_{2} \backslash N_{1}\right|=1,\left|N_{1} \backslash N_{2}\right|=0$ and $\left|N_{1} \cap N_{2}\right| \geq 3$. If $G\left[D_{2}\right] \cong \overline{K_{n-3}}$, then $\operatorname{diam}(G) \geq 3$, a contradiction. If $G\left[D_{2}\right] \cong K_{n-3}$ and $x_{1} x_{2} \in E$, then $G \cong H_{11}$. If $G\left[D_{2}\right] \cong K_{n-3}$ and $x_{1} x_{2} \notin E$, then let $y_{1}, y_{2}, y_{3} \in N_{1} \cap N_{2}$ and let $z \in N_{2} \backslash N_{1}$. Consider $S_{1}=\left\{u, y_{1}\right\}, S_{2}=\left\{x_{2}, y_{2}\right\}, S_{3}=\left\{z, y_{3}\right\}$ and let $\Pi=\left\{S_{1}, S_{2}, S_{3}\right\} \cup\{\{w\}$ : $\left.w \notin S_{1} \cup S_{2} \cup S_{3}\right\}$. Then, $\left\{x_{1}\right\}$ resolves both $S_{2}$ and $S_{3}$, and $S_{3}$ resolves $S_{1}$. It is easy to check that it is a dominating partition. Therefore, $\eta_{p}(G) \leq n-3$, a contradiction.

- $\left|N_{1} \cap N_{2}\right|=n-5$. Then, $\left|N_{2} \backslash N_{1}\right|+\left|N_{1} \backslash N_{2}\right|=2$ and $\left|N_{1} \cap N_{2}\right| \geq 2$. Let $y_{1}, y_{2} \in$ $\left(N_{2} \backslash N_{1}\right) \cup\left(N_{1} \backslash N_{2}\right)$ and $z_{1}, z_{2} \in N_{1} \cap N_{2}$, and let $S_{1}=\left\{y_{1}, z_{1}\right\}, S_{2}=\left\{y_{2}, z_{2}\right\}$ and $S_{3}=\left\{u, x_{1}\right\}$. Then, $\Pi=\left\{S_{1}, S_{2}, S_{3}\right\} \cup\left\{\{w\}: w \notin S_{1} \cup S_{2} \cup S_{3}\right\}$ is an RD-partition of $G$. Indeed, $S_{1}$ resolves $S_{3}$ and, for $i \in\{1,2\}, S_{i}$ is resolved by $S_{1}$ if $y_{i} \in N_{2} \backslash N_{1}$ and $S_{i}$ is resolved by $\left\{x_{2}\right\}$ if $y_{i} \in N_{1} \backslash N_{2}$. Besides, $\Pi$ is dominating. Hence, $\eta_{p}(G) \leq n-3$, a contradiction.

- $\left|N_{1} \cap N_{2}\right| \in\{n-6, n-7\}$. In such a case, $\left|N_{2} \backslash N_{1}\right|+\left|N_{1} \backslash N_{2}\right| \in\{3,4\}$. Since $\left|N_{2} \backslash N_{1}\right| \geq$ $\left|N_{1} \backslash N_{2}\right|$, we have $\left|N_{2} \backslash N_{1}\right| \geq 2$. Since $\operatorname{deg}\left(x_{1}\right) \geq n-3$, we have $\left|N_{1}\right| \geq n-5 \geq 2$. Let $y_{1}, y_{2} \in N_{1}$ and $z_{1}, z_{2} \in N_{2} \backslash N_{1}$. If $S_{1}=\left\{u, x_{1}\right\}, S_{2}=\left\{y_{1}, z_{1}\right\}$ and $S_{3}=\left\{y_{2}, z_{2}\right\}$, and $\Pi=\left\{S_{1}, S_{2}, S_{3}\right\} \cup\left\{\{w\}: w \notin S_{1} \cup S_{2} \cup S_{3}\right\}$, then $S_{1}$ resolves both $S_{2}$ and $S_{3}$, and $S_{2}$ resolves $S_{1}$. Moreover, $\Pi$ is a dominating partition. Therefore, $\eta_{p}(G) \leq n-3$, a contradiction.

Case 2: There exists at least one vertex $u$ of degree 1 and there is no vertex of degree 2. Since 
$\operatorname{diam}(G)=2$, the neighbor $v$ of $u$ satisfies $\operatorname{deg}(v)=n-1$. Let $\Omega$ be the set of vertices different from $v$ that are not leaves. Notice that there are at most two vertices of degree 1 in $G$, as otherwise all vertices in $\Omega$ would have degree between 3 and $n-4$, contradicting the assumption made at the beginning of the proof.

If there are exactly two vertices of degree 1 , then $|\Omega|=n-3$. In such a case, as for every vertex $w \in \Omega, \operatorname{deg}(w) \geq n-3, \Omega$ induces a complete graph in $G$, and hence $G \cong H_{13}$.

Suppose next that $u$ is the only vertex of degree 1 , which means that $\Omega$ contains $n-2$ vertices, all of them of degree $n-3$ or $n-2$. Consider the (non-necessarily connected) graph $J=\overline{G[\Omega]}$. Certainly, $J$ has $n-2$ vertices, all of them of degree either 0 or 1 . Let $L$ denote the set of vertices of degree 1 in $J$. Observe that the cardinality of $L$ must be even. We distinguish three cases.

- If $|L|=0$, then $G \cong K_{1} \vee\left(K_{1}+K_{n-2}\right)$, and by Theorem 15 we have $\eta_{p}(G)=n-1$, a contradiction.

- If $|L|=2$, then $G \cong H_{14}$.

- If $|L| \geq 4$, let $\left\{x_{1}, x_{2}, x_{3}, x_{4}\right\} \subseteq L$ such that $x_{1} x_{2}$ and $x_{3} x_{4}$ are edges of $J$, and let $y \in \Omega \backslash$ $\left\{x_{1}, x_{2}, x_{3}, x_{4}\right\}$. Consider the partition $\Pi=\left\{S_{1}, S_{2}\right\} \cup\left\{\{w\}: w \notin S_{1} \cup S_{2}\right\}$, where $S_{1}=\left\{v, x_{1}\right\}$, $S_{2}=\left\{u, x_{3}, y\right\}$. Observe that $\left\{x_{2}\right\}$ resolves $S_{1},\left\{u, x_{3}\right\}$ and $\{u, y\}$, and $\left\{x_{4}\right\}$ resolves $\left\{x_{3}, y\right\}$. Besides, $\Pi$ a is dominating partition. Therefore, $\eta_{p}(G) \leq n-3$, a contradiction.

\subsection{Case diameter 3}

We consider the case $\eta_{p}(G)=n-2$ and $\operatorname{diam}(G)=3$.

Proposition 21. Let $G$ be a graph of order $n \geq 7$ and diameter 3 . If $\eta_{p}(G)=n-2$, then $G \in\left\{H_{15}, H_{16}, H_{17}\right\}$ (see Figure 7).

Proof. By Lemma 13 (case $k=3$ ), every vertex has degree 1, 2, $n-3, n-2$ or $n-1$. Let $u$ and $v$ be two vertices such that $d(u, v)=3$. In such a case, both $u$ and $v$ have degree at most $n-3$.

Notice that on the one hand, it is not possible to have neither $\{\operatorname{deg}(u), \operatorname{deg}(v)\}=\{2, n-3\}$ nor $\{\operatorname{deg}(u), \operatorname{deg}(v)\}=\{n-3\}$, as otherwise we would have more than $n$ vertices because $N(u) \cap N(v)=$ $\emptyset$, a contradiction.

On the other hand, if $\operatorname{deg}(u)=\operatorname{deg}(v)=2$, then $\eta_{p}(G) \leq n-3$. Indeed, let $u x_{1} x_{2} v$ be a $(u, v)$-path and let $D_{i}=\{z: d(u, z)=i\}$, for $i \in\{1,2,3\}$. Since $\left|D_{1}\right|=2$, we may assume that $D_{1}=\left\{x_{1}, y_{1}\right\}$. If $\left|D_{2}\right| \geq 2$, let $y_{2} \in D_{2} \backslash\left\{x_{2}\right\}$. If $x_{1} y_{2} \in E$, let $S_{1}=\left\{x_{1}, x_{2}\right\}$ and $S_{2}=\left\{y_{1}, y_{2}, v\right\}$. If $x_{1} y_{2} \notin E$, then $y_{1} y_{2} \in E$, and consider $S_{1}=\left\{y_{1}, x_{2}\right\}$ and $S_{2}=\left\{x_{1}, y_{2}, v\right\}$. If $\left|D_{2}\right|=1$, then $v$ has a neighbor $z \in D_{3}$, so that $z$ must be also adjacent to $x_{2}$. Let $S_{1}=\left\{x_{1}, x_{2}, v\right\}$ and $S_{2}=\left\{y_{1}, z\right\}$. In all cases, $\Pi=\left\{S_{1}, S_{2}\right\} \cup\left\{\{w\}: w \notin S_{1} \cup S_{2}\right\}$ is an RD-partition, because it is dominating and $\{u\}$ resolves both $S_{1}$ and $S_{2}$. Hence, $\eta_{p}(G) \leq n-3$, a contradiction.

Therefore, we may assume that $\operatorname{deg}(u)=1$ and that every vertex at distance 3 from $u$ has degree 1,2 or $n-3$. Let $D_{i}=\{x \in V(G): d(u, x)=i\}$, for $i=1,2,3$. Thus, $\left|D_{1}\right|=1$. Let $D_{1}=\{w\}$. We distinguish cases, depending on the cardinality of $D_{3}$.

Case 1: $\left|D_{3}\right| \geq 3$. Then, $\operatorname{deg}(w) \leq n-4$, and therefore, $\operatorname{deg}(w)=2,\left|D_{1}\right|=\left|D_{2}\right|=1$ and $\left|D_{3}\right|=n-3 \geq 4$. Let $x$ be the only vertex in $D_{2}$. Notice that every vertex of $D_{3}$ is adjacent to $x$. We distinguish cases taking into account the degree of the vertices in $D_{3}$. 

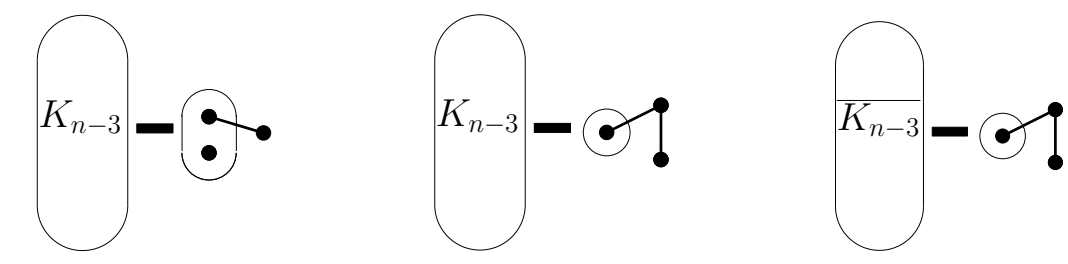

$H_{15} \cong H_{9}-e_{1}$

$H_{16} \cong H_{10}-e_{2}$

$H_{17} \cong H_{12}-e_{3}$

Figure 7: Graphs of order $n \geq 7$ and diameter 3 such that $\eta(G)=n-2$.

- There is a vertex of degree $n-3$ in $D_{3}$. A vertex in $D_{3}$ of degree $n-3$ must be adjacent to all the other vertices of $D_{3}$. Therefore, there is exactly one vertex of degree $n-3$ in $D_{3}$ or every vertex in $D_{3}$ has degree $n-3$. In the last case, that is, if every vertex in $D_{3}$ has degree $n-3$, then $D_{3}$ is a clique and $G \cong H_{16}$. Otherwise, let $y_{1}$ be the only vertex in $D_{3}$ of degree $n-3$. Any other vertex in $D_{3}$ has degree 2 , since it is adjacent to $x$ and to $y_{1}$. Let $y_{2}, y_{3}, y_{4} \in D_{3} \backslash\left\{y_{1}\right\}$. Consider $S_{1}=\left\{y_{1}, y_{2}\right\}$ and $S_{2}=\left\{w, x, y_{3}\right\}$. Then, $\Pi=\left\{S_{1}, S_{2}\right\} \cup\left\{\{z\}: z \notin S_{1} \cup S_{2}\right\}$ is an RD-partition of $G$. Indeed, it is dominating partition, $\{u\}$ resolves $S_{2}$ and $\left\{y_{4}\right\}$ resolves $S_{1}$ (see Figure 8(a)). Thus, $\eta_{p}(G) \leq n-3$, a contradiction.

- Every vertex in $D_{3}$ has degree 1 or 2, and at least one of them has degree 2. Then, $G\left[D_{3}\right]$ contains at least a copy of $K_{2}$. Let $y_{1}$ and $y_{2}$ be the vertices of such a copy of $K_{2}$, and take $y_{3} \in D_{3} \backslash\left\{y_{1}, y_{2}\right\}$. Consider $S_{1}=\left\{w, y_{1}\right\}, S_{2}=\left\{x, y_{2}\right\}$ and $S_{3}=\left\{u, y_{3}\right\}$. It is straightforward to check that $\Pi=\left\{S_{1}, S_{2}, S_{3}\right\} \cup\left\{\{z\}: z \notin S_{1} \cup S_{2} \cup S_{3}\right\}$ is an RD-partition of $G$ (see Figure 8(b)), and thus $\eta_{p}(G) \leq n-3$, a contradiction.

- Every vertex in $D_{3}$ has degree 1 . Then, $D_{3}$ induces an empty graph and $G \cong H_{17}$.

Case 2: $\left|D_{3}\right|=2$. Then, $\left|D_{2}\right|=n-4$. Let $D_{3}=\left\{y_{1}, y_{2}\right\}$. Recall that both $y_{1}$ and $y_{2}$ have at least a neighbor in $D_{2}$. We distinguish cases taking into account the degree of the vertices in $D_{3}$.

- There is a vertex of degree $n-3$ in $D_{3}$. We may assume that this vertex is $y_{1}$, and it must be adjacent to $y_{2}$ and to all vertices in $D_{2}$. So, there is a vertex $x_{1} \in D_{2}$ adjacent to both $y_{1}$ and $y_{2}$. Let $x_{2} \in D_{2} \backslash\left\{x_{1}\right\}$ and consider $S_{1}=\left\{w, x_{1}, y_{1}\right\}$ and $S_{2}=\left\{x_{2}, y_{2}\right\}$ Then, $\Pi=\left\{S_{1}, S_{2}\right\} \cup\left\{\{z\}: z \notin S_{1} \cup S_{2}\right\}$ is a dominating partition, and $\{u\}$ resolves both $S_{1}$ and $S_{2}$ (see Figure $8(\mathrm{c})$ ). Hence, $\eta_{p}(G) \leq n-3$, a contradiction.

- Both vertices in $D_{3}$ have degree 1 or 2 . Let $x_{1} \in D_{2}$ be a neighbor of $y_{1}$.

If there exists a vertex $x_{2} \in D_{2} \backslash\left\{x_{1}\right\}$ not adjacent to $y_{2}$, let $x_{3} \in D_{2} \backslash\left\{x_{1}, x_{2}\right\}$. Consider $S_{1}=\left\{w, x_{1}\right\}, S_{2}=\left\{x_{2}, y_{2}\right\}$ and $S_{3}=\left\{x_{3}, y_{1}\right\}$. Then, $\Pi=\left\{S_{1}, S_{2}, S_{3}\right\} \cup\left\{\{z\}: z \notin S_{1} \cup S_{2} \cup S_{3}\right\}$ is a dominating partition and $\{u\}$ resolves $S_{1}, S_{2}$ and $S_{3}$ (see Figure $8(\mathrm{~d})$ ). Therefore, $\eta_{p}(G) \leq$ $n-3$, a contradiction.

If all vertices in $D_{2} \backslash\left\{x_{1}\right\}$ are adjacent to $y_{2}$, then $\operatorname{deg}\left(y_{2}\right) \geq n-5$, with means that $2=$ $\operatorname{deg}\left(y_{2}\right)=n-5$ and thus $n=7$. Let $D_{2}=\left\{x_{1}, x_{2}, x_{3}\right\}$ and consider $S_{1}=\left\{w, x_{1}\right\}, S_{2}=\left\{x_{2}, y_{1}\right\}$ and $S_{3}=\left\{x_{3}, y_{2}\right\}$. Then, $\Pi=\left\{S_{1}, S_{2}, S_{3}\right\} \cup\left\{\{z\}: z \notin S_{1} \cup S_{2} \cup S_{3}\right\}$ is a dominating partition and $\{u\}$ resolves $S_{1}, S_{2}$ and $S_{3}$ (see Figure $8(\mathrm{e})$ ). Therefore, $\eta_{p}(G) \leq n-3$, a contradiction. 

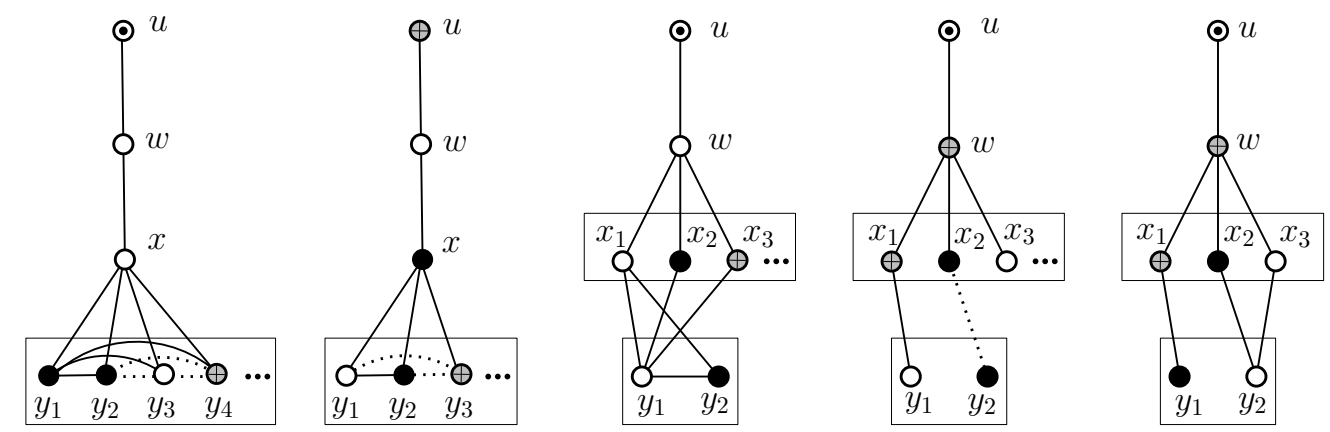

(a) $\left|D_{3}\right|=n-3$

(b) $\left|D_{3}\right|=n-3$

(c) $\left|D_{3}\right|=2$

(d) $\left|D_{3}\right|=2$

(e) $\left|D_{3}\right|=2$
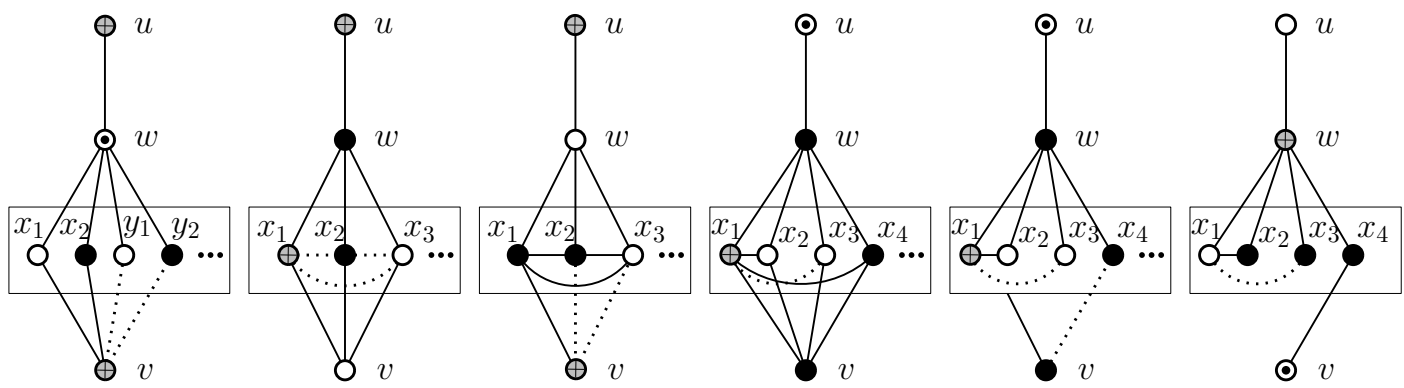

$$
\text { (f) }\left|D_{3}\right|=1 \quad(g)\left|D_{3}\right|=1
$$

(h) $\left|D_{3}\right|=1$

(i) $\left|D_{3}\right|=1$

(j) $\left|D_{3}\right|=1$

(k) $\left|D_{3}\right|=1$

Figure 8: Solid (resp. dotted) lines mean adjacent (resp. non-adjacent) vertices. Vertices with the same "color" belong to the same part.

Case 3: $\left|D_{3}\right|=1$. Then, $D_{3}=\{v\}$ and $\left|D_{2}\right|=n-3$. We distinguish cases taking into account the degree of $v$ and the subgraph induced by $D_{2}$.

- $\operatorname{deg}(v)=2$. Let $x_{1}$ and $x_{2}$ be the two neighbors of $v$, and take $y_{1}, y_{2} \in D_{2} \backslash\left\{x_{1}, x_{2}\right\}$. Let $S_{1}=\{u, v\}, S_{2}=\left\{x_{1}, y_{1}\right\}$ and $S_{3}=\left\{x_{2}, y_{2}\right\}$. Then, $\Pi=\left\{S_{1}, S_{2}, S_{3}\right\} \cup\{\{z\}: z \notin$ $\left.S_{1} \cup S_{2} \cup S_{3}\right\}$ is dominating partition such that $\{w\}$ resolves $S_{1}$, and $S_{1}$ resolves both $S_{2}$ and $S_{3}$ (see Figure 8(f)), implying that $\eta_{p}(G) \leq n-3$, a contradiction.

- $\operatorname{deg}(v) \in\{1, n-3\}$ and $D_{2}$ induces an empty graph.

If $\operatorname{deg}(v)=n-3$, let $x_{1}, x_{2}, x_{3} \in D_{2}$ and let $S_{1}=\left\{u, x_{1}\right\}, S_{2}=\left\{w, x_{2}\right\}$ and $S_{3}=\left\{v, x_{3}\right\}$. Then, $\Pi=\left\{S_{1}, S_{2}, S_{3}\right\} \cup\left\{\{z\}: z \notin S_{1} \cup S_{2} \cup S_{3}\right\}$ is a dominating partition such that $S_{1}$ resolves both $S_{2}$ and $S_{3}$, and $S_{3}$ resolves $S_{1}$ (see Figure $8(\mathrm{~g})$ ), implying that $\eta_{p}(G) \leq n-3$, a contradiction.

If $\operatorname{deg}(v)=1$, then $G \cong H_{17}$.

- $\operatorname{deg}(v) \in\{1, n-3\}$ and $D_{2}$ induces a complete graph.

If $\operatorname{deg}(v)=n-3$, then $G \cong H_{15}$.

If $\operatorname{deg}(v)=1$, let $x_{1} \in D_{2}$ be the neighbor of $v$ and $x_{2}, x_{3} \in D_{2} \backslash\left\{x_{1}\right\}$. Consider $S_{1}=\{u, v\}$, $S_{2}=\left\{w, x_{3}\right\}$ and $S_{3}=\left\{x_{1}, x_{2}\right\}$. Then, $\Pi=\left\{S_{1}, S_{2}, S_{3}\right\} \cup\left\{\{z\}: z \notin S_{1} \cup S_{2} \cup S_{3}\right\}$ is a 
dominating partition such that $S_{1}$ resolves both $S_{2}$ and $S_{3}$, and $S_{3}$ resolves $S_{1}$ (see Figure 8(h)), implying that $\eta_{p}(G) \leq n-3$, a contradiction.

- $\operatorname{deg}(v) \in\{1, n-3\}$ and $D_{2}$ induces neither a complete, nor an empty graph.

In that case, there exist vertices $x_{1}, x_{2}, x_{3} \in D_{2}$ such that $x_{1} x_{2} \in E(G)$ and $x_{1} x_{3} \notin E(G)$.

If $\operatorname{deg}(v)=n-3$, then $\operatorname{deg}\left(x_{1}\right) \geq 3$, and thus, $\operatorname{deg}\left(x_{1}\right) \geq n-3$. Hence, $x_{1}$ must be adjacent to any other vertex in $D_{2}$ different from $x_{3}$. Let $x_{4} \in D_{2} \backslash\left\{x_{1}, x_{2}, x_{3}\right\}$ and consider $S_{1}=\left\{w, x_{4}, v\right\}$ and $S_{2}=\left\{x_{2}, x_{3}\right\}$ Then, $\Pi=\left\{S_{1}, S_{2}\right\} \cup\left\{\{z\}: z \notin S_{1} \cup S_{2}\right\}$ is a dominating partition such that $\{u\}$ resolves $S_{1}$ and $\left\{x_{1}\right\}$ resolves $S_{2}$ (see Figure $8(\mathrm{i})$ ), implying that $\eta_{p}(G) \leq n-3$, a contradiction.

Finally, suppose that $\operatorname{deg}(v)=1$. If there is a leaf $x$ in $D_{2}$, then $d(u, v)=d(x, v)=3$. In such a case, interchanging the role of the vertices $u$ and $v$, the preceding cases for $\left|D_{3}\right| \geq 2$ apply and we are done. So, we can assume that any vertex in $D_{2}$ has degree at least 2 . Suppose that $v$ is not adjacent to some vertex $x_{4} \in D_{2} \backslash\left\{x_{1}, x_{2}, x_{3}\right\}$. Notice that such a vertex exists whenever $n \geq 8$, because $D_{2}$ has at least 5 vertices. Let $S_{1}=\left\{w, x_{4}, v\right\}$ and $S_{2}=\left\{x_{2}, x_{3}\right\}$. Then, $\Pi=\left\{S_{1}, S_{2}\right\} \cup\left\{\{z\}: z \notin S_{1} \cup S_{2}\right\}$ is a dominating partition such that $\{u\}$ resolves $S_{1}$ and $\left\{x_{1}\right\}$ resolves $S_{2}$. Therefore, $\Pi$ is an RD-partition of $G$ (see Figure $8(\mathrm{j})$ ), and so $\eta_{p}(G) \leq n-3$, a contradiction.

Finally, if $n=7$ and the only vertex $x_{4} \in D_{2} \backslash\left\{x_{1}, x_{2}, x_{3}\right\}$ is adjacent to $v$, take $S_{1}=\left\{x_{2}, x_{3}, x_{4}\right\}$ and $S_{2}=\left\{u, x_{1}\right\}$. Then, $\Pi=\left\{S_{1}, S_{2}\right\} \cup\left\{\{z\}: z \notin S_{1} \cup S_{2}\right\}$ is a dominating partition such that $\{v\}$ resolves both $\left\{x_{2}, x_{4}\right\}$ and $\left\{x_{3}, x_{4}\right\} ; S_{2}$ resolves $\left\{x_{2}, x_{3}\right\}$; and $S_{1}$ resolves $S_{2}$. Therefore, $\Pi$ is an RD-partition of $G$ (see Figure $8(\mathrm{k})$ ), and so $\eta_{p}(G) \leq n-3$, a contradiction.

As a straightforward consequence of Propositions 16, 19, 20 and 21, the following result is obtained.

Theorem 22. If $G$ is a graph of order $n \geq 7$, then $\eta_{p}(G)=n-2$ if and only if $G \in \Lambda_{n}$ (see Figure 3).

The solution for $\beta_{p}(G)=n-2$ is also almost immediately derived.

Theorem 23. If $G$ is a graph of order $n \geq 7$, then $\beta_{p}(G)=n-2$ if and only if $G \in \Lambda_{n} \backslash\left\{H_{12}, H_{17}\right\}$.

Proof. If $G \in \Lambda_{n} \backslash\left\{H_{12}, H_{17}\right\}$ then, according to Proposition 16, $\beta_{p}(G)=n-2$.

Conversely, let $G$ be a graph of order $n \geq 7$ such that $\beta_{p}(G)=n-2$. Thus, $\eta_{p}(G)=n-2$, since by Theorem 1 and Theorem 15 we know that $\beta_{p}(G) \geq n-1$ if and only if $\eta_{p}(G) \geq n-1$. Hence, by Theorem 22 , we derive that $G \in \Lambda_{n}$. Finally, $\beta_{p}(G)=n-3$ if $G \in\left\{H_{12}, H_{17}\right\}$. Indeed, in such a case, $\beta_{p}(G) \geq n-3$, because $G$ contains a twin set of cardinality $n-3$, and a resolving partition of cardinality $n-3$ for $H_{12}$ and $H_{17}$ is shown in Figure 9 .

Remark 24. Theorem 23 corrects an inaccurate result shown in [28] (Theorem 3.2).

A graph $G$ is called doubly-connected if both $G$ and its complement $\bar{G}$ are connected. We finally show a couple of Nordhaus-Gaddum-type results, which are a straightforward consequence of Theorems 22 and 23. 

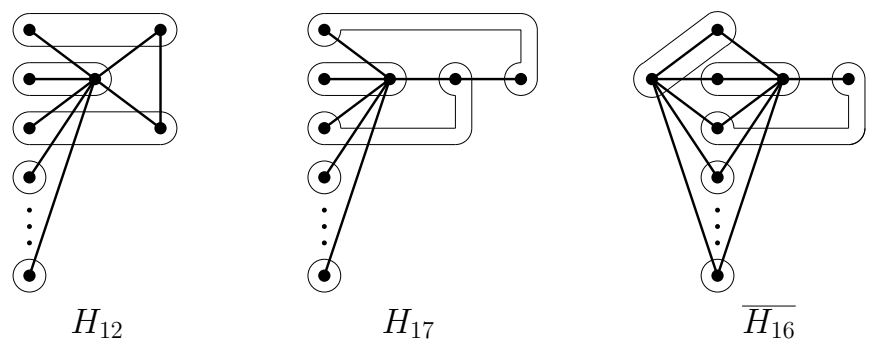

Figure 9: Resolving partitions of cardinality $n-3$ of $H_{12}, H_{17}$ and $\overline{H_{16}}$.

Theorem 25. If $G$ is a doubly-connected graph of order $n \geq 3$, then

(1) $6 \leq \eta_{p}(G)+\eta_{p}(\bar{G}) \leq 2 n-4$.

(2) The equality $\eta_{p}(G)+\eta_{p}(\bar{G})=6$ is attained, among others, by $P_{4}$ and $C_{5}$.

(3) If $n \geq 7$, then $\eta_{p}(G)+\eta_{p}(\bar{G})=2 n-4$ if and only if $G \in\left\{H_{15}, H_{17}\right\}$.

Proof. (1) Note that $\eta_{p}(G)=2$ if and only if $G \cong P_{2}$, but in this case $\bar{G}$ is not connected. Thus, if $G$ is a doubly-connected graph of order $n$, then $\eta_{p}(G) \geq 3$ and $\eta_{p}(\bar{G}) \geq 3$, and the lower bound holds. On other hand, by Theorem 15, if $\eta_{p}(G) \geq n-1$, then $\bar{G}$ is not connected. Thus, $\eta_{p}(G)+\eta_{p}(\bar{G}) \leq 2 n-4$.

(2) We know that $\overline{P_{4}}=P_{4}$ and $\overline{C_{5}}=C_{5}$, and it is easily verified that $\eta_{p}\left(P_{4}\right)=3$ and $\eta_{p}\left(C_{5}\right)=3$. Hence, $P_{4}$ and $C_{5}$ satisfy the given equality.

(3) Finally, a doubly-connected graph $G$ of order at least 7 attaining the upper bound must satisfy $\eta_{p}(G)=\eta_{p}(\bar{G})=n-2$. Therefore, the equality $\eta_{p}(G)+\eta_{p}(\bar{G})=2 n-4$ is attained if and only if $\{G, \bar{G}\} \subseteq\left\{H_{1}, \ldots, H_{17}\right\}$ (see Theorem 22). It is easy to check that this is satisfied if and only if $G \in\left\{H_{15}, H_{17}\right\}$ (observe that $\overline{H_{15}}=H_{17}$ ).

Theorem 26. If $G$ is a doubly-connected graph of order $n \geq 3$, then

(1) $4 \leq \beta_{p}(G)+\beta_{p}(\bar{G}) \leq 2 n-5$.

(2) $\beta_{p}(G)+\beta_{p}(\bar{G})=4$ if and only if $G=P_{4}$.

(3) If $n \geq 7$, then $\beta_{p}(G)+\beta_{p}(\bar{G})=2 n-5$ if and only if $G \in\left\{H_{15}, H_{16}, H_{17}\right\}$.

Proof. (1) Every graph $G$ of order at least 3 satisfies $\beta_{p}(G) \geq 2$. Hence, the lower bound holds. By Theorem 1, if a graph $G$ satisfies $\beta_{p}(G) \geq n-1$, then $\bar{G}$ is not connected. Therefore, any doubly-connected graph $G$ satisfies $\beta_{p}(G) \leq n-2$. By Theorem 23 , the graphs $G$ satisfying $\beta_{p}(G)=n-2$ are those from $\Lambda_{n} \backslash\left\{H_{12}, H_{17}\right\}$. It is easy to check that the only doubly-connected graphs of this set are $H_{15}$ and $H_{16}$. Their complements are $\overline{H_{15}}=H_{17}$, and $\overline{H_{16}}$ is shown in Figure 9. On the one hand, we have seen in the proof of Theorem 23 that $\beta_{p}\left(H_{17}\right)=n-3$. On the other hand, we have that $\beta_{p}\left(H_{16}\right)=n-3$. Indeed, $\beta\left(H_{16}\right) \leq n-3$ because $H_{16}$ has a twin set of cardinality $n-3$, and a resolving partition of cardinality $n-3$ is given in Figure 9 . Hence, $\beta_{p}(G)+\beta_{p}(\bar{G}) \leq 2 n-5$ if $G$ is doubly-connected. 
(2) We know that $\beta_{p}(G)=2$ if and only if $G$ is the path $P_{n}$, and $\overline{P_{n}}$ is a path if and only if $n=4$. Hence, the equality $\beta_{p}(G)+\beta_{p}(\bar{G})=4$ holds if and only if $G \cong P_{4}$.

(3) This equality is satisfied if and only if $G$ is a doubly-connected graph such that $\left\{\beta_{p}(G), \beta_{p}(\bar{G}\}=\right.$ $\{n-2, n-3\}$, and as we have seen in the proof of item i), it happens if and only if $G \in$ $\left\{H_{15}, H_{16}, H_{17}\right\}$.

Acknowledgement The authors are thankful to the anonymous referees for their valuable comments and remarks, which helped to improve the presentation of this paper.

\section{References}

[1] R. C. Brigham, G. Chartrand, R. D. Dutton and P. Zhang: Resolving domination in graphs. Math. Bohem., 128 (1) (2003), 25-36.

[2] J. Cáceres, C. Hernando, M. Mora, I. M. Pelayo and M. L. Puertas: On the metric dimension of infinite graphs. Discrete Applied Mathematics, 160 (18) (2012), 2618-2626.

[3] J. Cáceres, C. Hernando, M. Mora, I. M. Pelayo and M. L. Puertas: Locating-dominating codes: bounds and extremal cardinalities. Appl. Math. Comput., 220 (2013), 38-45.

[4] J. Cáceres, C. Hernando, M. Mora, I. M. Pelayo, M. L. Puertas, C. Seara and D. R. Wood: On the metric dimension of Cartesian products of graphs. SIAM J. Discrete Math., 21 (2) (2007), 423-441.

[5] G. Chartrand, L. Eroh, M. A. Johnson, and O. R. Oellermann: Resolvability in graphs and the metric dimension of a graph. Discrete Applied Mathematics, 105(1-3) (2000), 99-113.

[6] G. Chartrand, E. Salehi and P. Zhang: The partition dimension of a graph. Aequationes Mathematicae, 59 (2000), 45-54.

[7] G. G. Chappell, J. Gimbel and C. Hartman: Bounds on the metric and partition dimensions of a graph. Ars Combin., 88 (2008), 349-366.

[8] E. J. Cockayne and S. T. Hedetniemi: Towards a theory of domination in graphs. Networks, 7 (3) (1977), 247-261.

[9] M. Fehr, S. Gosselin and O. R. Oellermann: The partition dimension of Cayley digraphs. Aequationes Mathematicae, 71 (1-2) (2006), 1-18.

[10] W. Goddard: Mastermind revised. J. Combin. Math. Combin. Comput. Sci. , 51 (2004), 215-220.

[11] A. GonzÁlez, C. Hernando And M. Mora: Metric-locating-dominating sets of graphs for constructing related subsets of vertices. Appl. Math. Comput., 332 (2018), 449-456.

[12] I. González Yero, M. Jakovac, D. Kuziak and A. Taranenko: The partition dimension of strong product graphs and Cartesian product graphs. Discrete Math., 331 (2014), 43-52.

[13] C. Grigorious, S. Stephen, B. Rajan, M. Miller and A. William: On the partition dimension of a class of circulant graphs. Information Processing Letters, 114 (2014), 353-356. 
[14] F. Harary and R. Melter: On the metric dimension of a graph. Ars Combin., 2 (1976), 191-195.

[15] T. W. Haynes, M. A. Henning and J. Howard: Locating and total dominating sets in trees . Discrete Applied Mathematics, 154 (2006), 1293-1300.

[16] T. Haynes, D. Knisley, E. Seier And Y. Zou: A quantitative analysis of secondary RNA structure using domination based parameters on trees. BMC Bioinf., 7 (1) (2006), 108.

[17] M. A. Henning and O. R. Oellermann: Metric-locating-dominating sets in graphs. Ars Combin., 73 (2004), 129-141

[18] C. Hernando, M. Mora, I. M. Pelayo, C. Seara and D. R. Wood: Extremal graph theory for metric dimension and diameter. Electron. J. Combin., 17 (1) (2010), R30.

[19] C. Hernando, M. Mora And I. M. Pelayo: Nordhaus-Gaddum bounds for locating-domination. Eur. J. Combin., 36 (2014), 1-6.

[20] J. McCoy And M. A. Henning: Locating and paired-dominating sets in graphs. Discrete Applied Mathematics, 157 (15) (2009), 3268-3280.

[21] O. Ore: Theory of graphs. American Mathematical Society Colloquium Publications, 38. American Mathematical Society, Providence, R.I., (1962).

[22] J. A. Rodríguez-Velázquez, I. GonzÁlez Yero and M. Lemanska: On the partition dimension of trees. Discrete Applied Mathematics, 166 (2014), 204-209.

[23] V. Saenpholphat And P. Zhang: Conditional resolvability in graphs: a survey. International Journal of Mathematics and Mathematical Sciences, 38 (2004), 1997-2017.

[24] A. Sasireka and A. H. Nandhukishore: Applications of dominating set of graph in computer networks. Int. J. Eng. Sci. Res. Technol, 3 (1) (2014), 170-173.

[25] A. Sebo and E. Tannier: On metric generators of graphs. Math. Oper. Res., 29 (2) (2004), 383-393.

[26] P. J. Slater: Leaves of trees. Proc. 6th Southeastern Conf. on Combinatorics, Graph Theory, and Computing, Congr. Numer., 14 (1975), 549-559.

[27] S. Stephen, B. Rajan, C. Grigorious and A. William. : Resolving-power dominating sets. Appl. Math. Comput., 256 (2015), 778-785.

[28] I. Tomescu: Discrepancies between metric dimension and partition dimension of a connected graph. Discrete Math., 308 (2008), 5026-5031. 